\title{
Atypische Beschäftigung im Erwerbsverlauf: Verlaufstypen als Ausdruck sozialer Spaltung? \\ Atypical Employment and Careers: Do Types of Careers Reflect Social Division?
}

\section{Petra Böhnke}

Universität Hamburg, Fakultät Wirtschafts- und Sozialwissenschaften, Welckerstr. 8, D-20354 Hamburg, Germany Petra.Boehnke@wiso.uni-hamburg.de

\section{Janina Zeh}

Universität Hamburg, Fakultät Wirtschafts- und Sozialwissenschaften, Welckerstr. 8, D-20354 Hamburg, Germany Janina.Zeh@wiso.uni-hamburg.de

\section{Sebastian Link}

Universität Hamburg, Fakultät Wirtschafts- und Sozialwissenschaften, Welckerstr. 8, D-20354 Hamburg, Germany Sebastian.Link@wiso.uni-hamburg.de

Zusammenfassung: Der Artikel beleuchtet die Dynamik atypischer Beschäftigung in individuellen Erwerbsverläufen auf der Grundlage des Sozio-oekonomischen Panels 2002-2011. Mit Hilfe von Sequenzmuster- und Clusteranalysen werden Erwerbsverläufe in unterschiedlichen biografisch bedeutsamen Erwerbsphasen typisiert. Insbesondere Erwerbsverläufe in der beruflichen Einstiegsphase integrieren atypische Beschäftigung episodenhaft. In der Haupterwerbsphase tritt atypische Beschäftigung verstärkt in kontinuierlicher Form auf und wird von Frauen in unbefristeter Teilzeit dominiert. Dies gilt - weniger weit verbreitet - auch für den Übergang in die Rente. Es lassen sich phasen-, geschlechts- und qualifikationsspezifische Verlaufsmuster mit atypischer Beschäftigung erkennen, oftmals ohne Berührungspunkte mit dem Normalarbeitsverhältnis. Diese Spaltungstendenzen bringen aber nur für wenige Betroffene eine Kumulation von Risiken sozialer Ungleichheit mit sich.

Schlagworte: Arbeitsmarkt; Atypische Beschäftigung; Erwerbsverlauf; Soziale Spaltung; Soziale Ungleichheit; Erwerbsphasen; Bildung; Gender; Sequenzanalyse; Clusteranalyse; SOEP.

Summary: This article examines the role of atypical employment in individual careers. Using sequence and cluster analyses based on data from the German Socio-Economic Panel from 2002 to 2011, it identifies career types in three biographically relevant employment phases. Employment careers integrate atypical employment in particular during the job-entry phase, which, usually, is episodic in nature. During the main phase of employment, atypical employment turns out to be more stable over time and is mainly found among women who work permanently in part-time jobs. This also applies - though less wide-spread - for the transition into retirement. We can identify phase-, gender-, and qualification-specific career types that integrate atypical employment, often with hardly any points of contact to standard employment. These socially divisive tendencies, however, entail an accumulation of social risks for relatively few people.

Keywords: Labor Market; Atypical Employment; Employment Careers; Social Division; Social Inequality; Employment Phases; Qualification; Gender; Sequence Analysis; Cluster Analysis; GSOEP.

\section{Einleitung ${ }^{1}$}

Der Anteil atypisch Beschäftigter an den abhängig Erwerbstätigen ist seit den 1970er Jahren in Deutsch-

${ }^{1}$ Wir danken dem Herausgebergremium der Zeitschrift für Soziologie und zwei anonymen GutachterInnen für ihre wertvollen Kommentare. Weiterer Dank gilt den Teil- land kontinuierlich gestiegen (Keller \& Seifert 2011; Schmeißer et al. 2012). Mit der Zunahme atypischer Beschäftigungsverhältnisse werden weitrei-

nehmerInnen der Tagung „Die gespaltene Gesellschaft“ an der Johannes Kepler Universität in Linz sowie der „SOEP User Conference 2014“ in Berlin für ihre anregenden Diskussionsbeiträge. 
chende Verunsicherungen im Hinblick auf Lebensstandard, Planbarkeit und soziale Integration assoziiert (Brinkmann et al. 2006; Castel \& Dörre 2009; Mayer-Ahuja 2003; Vogel 2006). So ist mit Verweis auf die disziplinierende Wirkung atypischer Beschäftigung von einer bis in die gesellschaftliche Mitte hineinreichenden Verunsicherung die Rede (Bourdieu 1998), andernorts von einer Polarisierung der Arbeiterschaft in sichere Kern- und der Prekarität ausgesetzten Randbelegschaften (Bude 2009). Ob sich Prekarität unweigerlich ausbreitet und „sozialstrukturelle Eindeutigkeiten“ dabei auflöst (Vogel 2006: 76), oder ob sich atypische Beschäftigung in bestimmten Erwerbsverläufen konzentriert (Bartelheimer 2011: 391) und damit soziale Spaltung befördert, ist noch nicht zufriedenstellend beantwortet.

Eine empirische Untermauerung der einen oder anderen Position setzte Kenntnis darüber voraus, bei wem, wie häufig, mit welcher Dauer und in welchem erwerbsbiografischen Kontext atypische Beschäftigung auftritt. Es ist die Heterogenität der atypischen Beschäftigungsformen (Befristung, Geringfügigkeit, Leiharbeit, Teilzeit) und der atypisch Beschäftigten, die die Forschungslage in diesem Themenfeld unübersichtlich macht.

Atypische Beschäftigung kann in ihren einzelnen Formen je Unterschiedliches bedeuten: Sie kann Arbeitslosigkeit beenden oder die Notwendigkeit, Familie und Beruf zu vereinbaren, widerspiegeln. Sie kann gezielt zum Sammeln von Berufserfahrung eingesetzt und als Grundlage für zukünftigen beruflichen Aufstieg gedacht sein. Atypische Beschäftigung steht auch für Instabilität, Ausgrenzung und Sackgasse, wenn sie die Existenz nicht sichert und keine langfristige Perspektive bietet. Eine systematische verlaufsorientierte Analyse darüber, wie atypische Beschäftigung in ihren einzelnen Formen in Erwerbsbiografien eingebunden ist und für wen bestimmte Verläufe besonders wahrscheinlich sind, steht aber noch aus.

Es fehlen sozialstrukturell rückgebundene Einsichten in die Dynamik von Erwerbsverläufen, die von atypischer Beschäftigung geprägt sind. Noch lassen sich keine Aussagen darüber treffen, ob mit der Ausweitung atypischer Beschäftigung unweigerlich eine Destandardisierung von Erwerbsbiografien einhergeht und die Anzahl der Episoden in atypischer Beschäftigung für alle Erwerbstätigen gleichermaßen steigt. Ebenso ist bislang nicht hinreichend empirisch untermauert worden, ob sich atypische Beschäftigung in bestimmten Lebens- und Erwerbsphasen konzentriert (Diewald 2004; Ha- cket 2012) und ob dies in Abhängigkeit von soziodemografischen Merkmalen der betroffenen Personen geschieht. Erkenntnisse darüber versprächen aber Aufschluss über eventuell vorliegende soziale Spaltungsprozesse, die sich erwerbsverlaufsbezogen Geltung verschaffen. Daraus resultiert unsere Forschungsfrage mit explorativem Charakter: Welche Typen individueller Verlaufsmuster mit atypischer Beschäftigung lassen sich ausmachen und wie unterscheiden sie sich je nach erwerbsbiografischer Phase und sozio-demografischen Merkmalen der Beschäftigten?

Zunächst schildern wir den Forschungsstand zu atypischer Beschäftigung unter besonderer Berücksichtigung der Übergangs- und Verlaufsperspektive. Aus Überlegungen zu institutionellen Rahmenbedingungen von Erwerbsverläufen sowie zu genderund qualifikationsspezifischen Ausdifferenzierungen leiten wir Annahmen zu Verlaufsmustern und dem jeweiligen Stellenwert atypischer Beschäftigung in der beruflichen Einstiegs-, Haupt- und Endphase von Männern und Frauen ab. Mithilfe von Sequenzmuster- und Clusteranalysen auf der Grundlage von Daten des Sozio-oekonomischen $\mathrm{Pa}$ nels (SOEP) der Jahre 2002 bis 2011 zeigen wir im empirischen Teil Erwerbsverlaufsmuster auf und beschreiben ihre sozio-demografischen Merkmale. Die Ergebnisse sollen die Diskussion darum bereichern, ob es vermehrt Verläufe mit atypischer Beschäftigung gibt, die im Sinne einer Risikoakkumulation Merkmale sozialer Ungleichheit auf sich vereinen und damit gesellschaftlichen Spaltungstendenzen Ausdruck verleihen.

\section{Forschungsstand und theoretische Einordnung}

Atypische Beschäftigung wird in Abgrenzung vom Normalarbeitsverhältnis definiert, d. h. in Bezug zu den gängigen Normalitätsstandards abhängiger Erwerbsarbeit (Rademacher \& Ramos Lobato 2008: 124f.). Damit ist in der Regel eine unbefristete Stelle in Vollzeit gemeint, die zudem mit einer vollständigen Integration in die sozialen Sicherungssysteme, der Identität des Arbeits- und Beschäftigungsverhältnisses und der Weisungsgebundenheit des Arbeitnehmers einhergeht (Keller \& Seifer 2011: 8, unter Rückbezug auf Mückenberger 1985, 2010). $\mathrm{Zu}$ den atypischen Beschäftigungsformen zählen Teilzeitarbeit, geringfügige Beschäftigung (MiniJobs), Midi-Jobs, befristete Beschäftigung, Leiharbeit und neue Formen der Selbstständigkeit (z. B. 
Ich- bzw. Familien-AGs) (Keller \& Seifert 2011: 10ff.). ${ }^{2}$

Der Anteil atypisch Beschäftigter an allen Erwerbstätigen belief sich im Jahr 2012 auf 21,8 \% (Statistisches Bundesamt 2013). Insbesondere junge Menschen, Migrantinnen und Migranten, Frauen, Alleinerziehende und gering Qualifizierte sind atypisch beschäftigt, lediglich Leiharbeit ist männlich dominiert (Brinkmann et al. 2006: 19ff.; Keller \& Seifert 2011: 13ff.; Lengfeld \& Kleiner 2009: 47ff.; Fromm \& Bartelheimer 2012: 11; Giesecke 2009; Schmeißer et al. 2012: 29ff.; Statistisches Bundesamt 2008; Weinkopf et al. 2009). Der im Folgenden dargestellte Forschungsstand sowie unsere eigenen Analysen beziehen sich ausschließlich auf abhängig Beschäftigte.

\subsection{Forschungsstand zu atypischer Beschäftigung aus der Übergangs- und Verlaufsperspektive}

Ein Großteil der empirischen Forschung zu atypischer Beschäftigung im Zeitverlauf konzentriert sich auf Übergänge zwischen Erwerbsverhältnissen. Befristete Beschäftigung führt sowohl in Nichterwerbstätigkeit als auch in Normalarbeit und hat verglichen mit anderen atypischen Formen ambivalenten Charakter (Brzinsky-Fay et al. 2007: 16f.). Im Vergleich zu Teilzeitbeschäftigung und Leiharbeit macht Befristung den Übergang in ein Normalarbeitsverhältnis, d.h. einen Brückeneffekt, wahrscheinlicher. Geringfügige Beschäftigung leitet am häufigsten in Nichterwerbstätigkeit über (Brülle 2013; Brzinsky-Fay et al. 2007: 17). Der Brückeneffekt befristeter Beschäftigung ist insbesondere für hoch Qualifizierte zu beobachten (Gundert \& Hohendanner 2013: 3). Zudem zeigt sich für alle atypischen Beschäftigungsverhältnisse, dass höheres Alter die Chance auf einen Übergang in ein Normalarbeitsverhältnis reduziert (Brülle 2013: 167; Gensicke et al. 2010: 184f.). Für Frauen ist die Übergangswahrscheinlichkeit in ein Normalarbeitsverhältnis geringer als für Männer (Brzinsky-Fay et

\footnotetext{
2 Verschiedene Formen staatlich geförderter Beschäftigung wie Arbeitsbeschaffungsmaßnahmen (ABM) und Ein-Euro-Jobs gehören somit nicht dazu. In der deutschen Forschung gelten ABMs und Ein-Euro-Jobs mehrheitlich nicht als atypische Beschäftigung (siehe z. B. Keller \& Seifert 2011: 9; Sachverständigenrat zur Begutachtung der gesamtwirtschaftlichen Entwicklung 2008: 423ff.; Weinkopf et al. 2009: 15ff.), was sich unter anderem damit begründen lässt, dass es sich um eine mit öffentlichen Geldern finanzierte Zusatzbeschäftigung handelt, für die kein Lohn, sondern eine Aufwandsentschädigung gezahlt wird.
}

al. 2007: 16; Gensicke et al. 2010: 185), insbesondere wenn Kinder im Haushalt leben (Brülle 2013: 167). In der Übergangsforschung spielen Muster der Einbettung von atypischer Beschäftigung in Erwerbsverläufe eine untergeordnete Rolle, weil sie sich hauptsächlich mit der Frage beschäftigt, ob atypische Beschäftigung in ein Normalarbeitsverhältnis überführt werden kann.

Werden Erwerbsverläufe in den Blick genommen, zeigt sich im Kohortenvergleich, dass Diskontinuität vor allem in der Einstiegs- und Endphase bedeutsamer wird. Beispielsweise erfolgt der Übergang von Erwerbstätigkeit in Rente häufiger im Anschluss an atypische Beschäftigung in Form von Teilzeit oder Geringfügigkeit, dies insbesondere für Frauen und niedrig Qualifizierte (Ebert \& Trischler 2012: 538ff.). Jedoch besteht in der beruflichen Einstiegs-, Haupterwerbs- und Endphase das Muster kontinuierlicher Vollerwerbstätigkeit mehrheitlich fort und das Risiko von Arbeitslosigkeit oder atypischer Beschäftigung konzentriert sich in davon separaten Verlaufsmustern. Insbesondere Mutterschaft führt zu einer weniger intensiven und häufig diskontinuierlichen Erwerbsbeteiligung (Bartelheimer 2011; Ebert \& Trischler 2012; Hacket 2012; Schmidt 2012). Grimm und Vogel (2010) typisieren prekarisierte Erwerbsbiografien anhand qualitativer Paneldaten und identifizieren neue Arbeitsmarktakteure, die sie als erwerbsgesellschaftliche Grenzgänger bezeichnen. Dazu zählen sie jene, die zwischen Perioden der Arbeitslosigkeit und Erwerbstätigkeit wie auch zwischen unterschiedlich gut gesicherten Erwerbsformen pendeln (Grimm \& Vogel 2010: 6f.). Diese Ergebnisse verweisen darauf, dass atypische Beschäftigung auch episodenhaft in einer ganzen Reihe von Erwerbsverläufen eine Rolle spielen kann.

Insgesamt zeigt sich, dass atypische Beschäftigung in der Analyse von Erwerbsverläufen nur selten explizit berücksichtigt wird. Die wenigen Studien, die dazu vorliegen, beziehen sich häufig nur auf einzelne Formen (siehe z. B. Klenner \& Schmidt 2011, 2012). Zudem liefern sie kaum Erkenntnisse darüber, ob atypische Beschäftigung in einem spezifischen Zusammenhang mit erwerbsbiografischen und sozio-demografischen Merkmalen der Beschäftigten steht. Hier setzt unser Beitrag an. Wir fragen, in welchen Verläufen atypische Beschäftigung in welcher Weise auftritt und von wem sie vorrangig ausgeübt wird. Damit rückt eine längere und holistische Verlaufsgeschichte in den Fokus, die über punktuelle Übergangswahrscheinlichkeiten und Momentaufnahmen hinausgeht. So lässt sich zeigen, was sich vor und nach möglichen Übergängen ab- 
spielt, wie lange bestimmte Zustände andauern und mit welcher Frequenz es zu Wechseln kommt. Die von uns betrachteten Erwerbsverlaufsmuster, die Auszüge aus Gesamtverläufen darstellen, sind somit als eine „Art Scharnier zwischen einzelnen Übergängen und gesamten Verläufen“ (Sackmann \& Wingens 2001: 32) zu verstehen.

Ausgehend vom geschilderten Forschungsstand sind spezifische Verlaufsmuster zu vermuten, in denen eine atypische Erwerbseinbindung eine Rolle spielt: Wechsel von atypischer Beschäftigung sowohl in Arbeitslosigkeit und Nichterwerbstätigkeit als auch in reguläre Beschäftigung, konstant atypische sowie episodenhaft atypische Verläufe. Unter Letzterem verstehen wir einen Verlauf, der durch Wechsel zwischen verschiedenen Erwerbsstatus und (mehrmaligem) Einmünden in atypische Beschäftigung geprägt ist. Inwiefern diese Vermutungen dem empirischen Abgleich mit komplexen Erwerbsverläufen standhalten, gilt es in unserem Beitrag zu prüfen. Um zu Annahmen über Verlaufstypen in verschiedenen Erwerbsphasen und ihre sozio-demografische Rückbindung zu kommen, gehen wir im Folgenden auf die institutionellen Rahmenbedingungen von Erwerbsverläufen sowie auf ihre gender- und qualifikationsspezifische Ausdifferenzierung ein.

\subsection{Institutionelle Rahmenbedingungen}

Personen, die ihre Arbeitskraft auf dem Arbeitsmarkt anbieten, treffen auf jeweils spezifische institutionelle und historische Kontexte - sowohl zum Zeitpunkt ihres Erwerbseinstiegs als auch in ihrem weiteren Erwerbsverlauf. Die Wahrscheinlichkeit, atypisch beschäftigt zu sein, variiert mit diesen Rahmenbedingungen, aber auch mit dem biografischen Zeitpunkt, an dem sie für die jeweiligen Kohorten relevant werden. Die letzten Jahrzehnte werden mit einer „Zunahme berufsbiographischer Erfahrungen von Unstetigkeit und Unsicherheit" (Diewald \& Eberle 2003: 13) assoziiert. Dem voraus ging die Phase des „Fordismus“, die in der Lebensverlaufsforschung als eine Phase der Institutionalisierung und Standardisierung des Lebens- und Erwerbsverlaufs beschrieben wird, in der sich das Normalarbeitsverhältnis, gekoppelt an ein männliches Alleinernährermodell, als Referenzkategorie für Ansprüche an Kontinuität und Sicherheit im Berufsleben herausbilden konnte.

Ab Mitte der 1970er Jahre etablierten sich neue Formen von Arbeitsorganisation, die einen Schwerpunkt auf Flexibilisierung setzten und eine wach- sende Randbelegschaft mit sich brachten (MayerAhuja et al. 2012). Die zunehmende Pluralisierung von Erwerbsverläufen kann dabei zum Teil auch als Ausdruck veränderter Lebensweisen, (Sinn-)Ansprüche sowie Erwartungen an Erwerbsarbeit von Individuen bzw. Haushalten betrachtet werden (Baethge 1991; Grunow et al. 2005). Der Flexibilisierungstrend verstärkte sich infolge der ab 2002 implementierten Gesetze für moderne Dienstleistungen am Arbeitsmarkt. Ein wichtiges Ziel war dabei die Senkung der Arbeitslosenzahlen und die Verkürzung der Arbeitslosigkeitsdauer. Vor diesem Hintergrund verlor das Ziel, Arbeitslose vorrangig in eine sozial gesicherte Beschäftigung zu vermitteln, an Bedeutung (Oschmiansky et al. 2007: 292ff.). Durch die neue Gesetzeslage kam es zu einer verstärkten Förderung atypischer Beschäftigung, insbesondere von Leiharbeit, Mini- und Midijobs (Seifert 2005: 19f.).

Arbeitsmarktpolitische Maßnahmen gingen also einher mit wirtschaftlichem Wandel und Individualisierungstendenzen. Dieses Zusammenspiel machte atypische Beschäftigung sowohl auf der Angebotsals auch auf der Nachfrageseite wahrscheinlicher. Im Hinblick auf unterschiedliche Erwerbseinstiegskohorten erscheint es aber plausibel, von erwerbsphasenspezifisch ausgeprägten Erwerbsverlaufsmustern auszugehen, die atypische Beschäftigung auf besondere Weise integrieren. Trotz Flexibilisierungsmaßnahmen fungiert das Normalarbeitsverhältnis in den verschiedenen Politikbereichen weiterhin als normative Orientierungsgrundlage (Giesecke 2006: 57f.), und die Normalarbeitsbiografie befindet sich nicht in Auflösung. Dies gilt insbesondere für Personen, die sich derzeit am Ende ihrer Erwerbsbiografie befinden. Sie traten in Zeiten in den Arbeitsmarkt ein, in denen sie noch von Schutzmechanismen profitierten, die sich häufig an Senioritätsregeln orientierten. Im Vergleich dazu sind Personen, die sich derzeit in der beruflichen Einstiegsphase befinden oder einen Wiedereinstieg in den Arbeitsmarkt bewältigen müssen, stärker von flexibilisierten Arbeitsmarktbedingungen betroffen.

Konkret gehen wir davon aus, dass atypische Beschäftigung für Personen in der Einstiegsphase im Vergleich zu Personen in der Haupt- und Endphase eine größere Rolle in dem beobachteten Verlauf spielt (Bukodi et al. 2006), mehrheitlich als Wechsel von befristeter in reguläre Beschäftigung oder episodenhaft im Sinne eines Wechsels zwischen Arbeitslosigkeit und den in den letzten Jahren verstärkt geförderten atypischen Formen der Geringfügigkeit und Leiharbeit. Hier sind qualifikations- 
spezifische Unterschiede zu erwarten: Einerseits ist denkbar, dass die Zunahme schulischer Ausbildungen und Hochschulabschlüsse vermehrt eine praktische Einarbeitungs- und Erprobungsphase nach sich ziehen, die über Befristungen realisiert wird. Andererseits werden möglicherweise Personen ohne Ausbildungsabschluss oder mit niedriger Qualifikation in atypische Erwerbsformen wie bspw. Minijobs oder Leiharbeit abgedrängt, weil formale Bildungsabschlüsse für ein Normalarbeitsverhältnis immer wichtiger werden. Dieser durch die Bildungsexpansion angestoßene institutionelle Wandel spricht für stärker von atypischer Beschäftigung geprägte Erwerbsverläufe von Personen in der Einstiegsphase.

Ebenfalls plausibel und im Einklang mit dem in 2.1 präsentierten Forschungsstand ist, dass die letzten Jahre der Erwerbsbiografie durch mehr Atypik gekennzeichnet sind als die Haupterwerbsphase, was beispielsweise über veraltete Qualifikationen, technologischen Wandel, schlechtere Gesundheit und (freiwillige oder unfreiwillige) Altersteilzeit erklärbar ist. ${ }^{3}$ Dies verweist darauf, dass Alters- und Kohorteneffekte in verschiedene Richtungen weisen können. Für die Haupterwerbsphase, die eine Zeit der Stabilisierung bzw. Verfestigung und des Aufstiegs darstellt, erwarten wir mehrheitlich konstante Verläufe, sowohl im Hinblick auf reguläre Beschäftigungsverhältnisse als auch auf atypische Beschäftigungsformen wie unbefristete Teilzeit. Hier ist mit klaren genderspezifischen Ausdifferenzierungen zu rechnen, da die Haupterwerbsphase für Frauen häufig durch Anforderungen an die Vereinbarkeit von Elternschaft und Beruf geprägt ist.

\subsection{Gender- und qualifikationsspezifische Ausdifferenzierung}

Daran anknüpfend versprechen Überlegungen $z u$ genderspezifischen Ungleichheiten zusätzliche Einsichten. Atypische Beschäftigungsverhältnisse stellen keine neue Erscheinung dar, sondern gehören zur Institution des „weiblichen Lebenslaufs“ (Völker 2009: 274). Erwerbsverläufe von Frauen folgen nicht der klassischen Dreiteilung in Ausbildungs-, Erwerbs- und Rentenphase, sondern sind stärker von Erwerbsunterbrechungen und Wiedereinstiegen geprägt. Atypische Beschäftigung als Hinzuverdienst und Vereinbarkeitsmodus in Form von Teil-

\footnotetext{
${ }^{3}$ Im Gegensatz zu Teilzeit kommen Befristung und Leiharbeit vergleichsweise selten bei älteren Arbeitnehmern vor (siehe z. B. Schmeißer et al. 2012: 33; IG Metall Vorstand 2012: 49).
}

zeit oder geringfügiger Beschäftigung ist folglich als Bestandteil weiblicher Erwerbsbiografien in allen hier betrachteten Erwerbsphasen zu berücksichtigen.

Wir vermuten aber eine unterschiedlich starke Betroffenheit nach Erwerbseinstiegskohorten. Heute zeichnen sich typische Erwerbsmuster von Frauen durch eine höhere Erwerbsbeteiligung aus, was sowohl auf veränderte Rollenvorstellungen als auch auf ökonomische Notwendigkeiten zurückzuführen ist (Klenner \& Schmidt 2012: 22). Immer mehr Frauen besitzen eine klare berufliche Orientierung (Allmendinger 2009), sorgen für sich selbst und sind besser ausgebildet. Frauen in Paarhaushalten können sich wegen der tendenziell instabileren Erwerbsverläufe von Männern und aufgrund gestiegener Trennungsrisiken weniger auf eine lebenslange finanzielle Absicherung durch einen Partner verlassen (Klenner \& Schmidt 2012: 22). Jedoch sind „typisch weibliche Verläufe" nach wie vor stärker durch Unterbrechungen und atypische Beschäftigungsformen und weniger durch Aufstiege gekennzeichnet (Kohli 2003: 531). Der größte Teil des Zuwachses der Erwerbsbeteiligung speist sich aus Teilzeitarbeit und geringfügiger Beschäftigung (Schmeißer et al. 2012), wobei geringfügige Beschäftigung nicht nur von Frauen in Partnerschaften im Rahmen stabiler Teilzeitverläufe ausgeübt wird. Beide Formen spielen auch innerhalb diskontinuierlicher Erwerbsverläufe eine Rolle und sind in der Lebensverlaufsperspektive riskant, weil sie Altersarmut nach sich ziehen (Klenner \& Schmidt 2011, 2012). In den dargestellten Entwicklungen der weiblichen Erwerbsbeteiligung spiegelt sich die Tatsache, dass auf politischer Ebene neben defamilialisierenden auch dekommodifizierende und (re)familialisierende Bemühungen koexistieren, so z. B. die Regelungen zur Bedarfsgemeinschaft, zum Ehegattensplitting oder Betreuungsgeld (Scherschel \& Booth 2012: 24f.).

Zusammenfassend nehmen wir an, dass es insbesondere Frauen sind, die sich in Verläufen finden, die durch einen Wechsel von atypischer Beschäftigung in Nichterwerbstätigkeit geprägt sind - in der Einstiegsphase aufgrund von Mutterschaft, in der Endphase als Übergang in die Rente. Ebenfalls überrepräsentiert vermuten wir sie in der Einstiegsund Haupterwerbsphase in konstant oder episodenhaft atypischen Verläufen, wobei ein konstant atypischer Verlauf in unbefristeter Teilzeit in der Haupterwerbsphase sicherlich dominiert. Diese Annahmen gelten insbesondere für Frauen in Paarhaushaushalten und bzw. oder Frauen mit Kindern. 
Weiterhin sind Überlegungen zu Erwerbsverlaufsdifferenzen nach Bildungsniveau bzw. Qualifikation aufschlussreich: Den Ergebnissen aus Übergangsstudien folgend ist der bereits genannte Brückeneffekt befristeter Beschäftigung insbesondere für Hochqualifizierte zu beobachten (Gundert \& Hohendanner 2013: 3). Befristete Beschäftigung wird von Unternehmen vor allem dann im Sinne einer verlängerten Probezeit genutzt, wenn die betreffenden Positionen komplexe Tätigkeiten beinhalten und eine Bewertung der Produktivität erschwert möglich ist (Brülle 2013: 161). Die Ausübung komplexer Tätigkeit wiederum impliziert eine hohe Qualifikation. Geringe Qualifikationen oder ein fehlender Ausbildungsabschluss hingegen machen eine Beschäftigung in Arbeitsmarktsegmenten wahrscheinlich, die von schnellen Anlernphasen, dem Merkmal der Austauschbarkeit und wenig Investition in Betriebsbindung von Arbeitgeberseite gekennzeichnet sind (Sengenberger 1987). Die Beschäftigungsfähigkeit der Erwerbsperson wird so zwar aufrechterhalten, aber nicht maßgeblich verbessert, was den Verbleib in atypischer Beschäftigung oder auch einen Wechsel in Arbeitslosigkeit wahrscheinlich macht. Für das Verbleiben in atypischer Beschäftigung sprechen insbesondere bei Geringqualifizierten deren vergleichsweise kleine und homogene persönliche Netzwerke (Mewes 2010), die auf geringere Chancen verweisen, Netzwerkstrukturen zur Informationsgewinnung und somit als Jobmotor zu nutzen (Granovetter 1995).
Wir nehmen deshalb an, dass sich vor allem geringqualifizierte Personen in episodenhaft oder konstant atypischen Erwerbsverläufen befinden, ebenso in Verläufen, die durch Abstiege von atypischer Beschäftigung in Arbeitslosigkeit geprägt sind. Atypische Beschäftigung wird hier vermutlich vorrangig in Form von Leiharbeit und insbesondere geringfügiger Beschäftigung auftreten, da Letztere häufig die Funktion übernimmt, günstige Arbeitskraft für unspezifische Aufgaben bereitzustellen (Brülle 2013).

\subsection{Annahmen}

Die in den vorangegangenen Abschnitten entwickelten Annahmen bezüglich der Frage, in welchen Verläufen atypische Beschäftigung in welcher Weise auftritt und von wem sie vorrangig ausgeübt wird, sind in Tabelle 1 zusammengefasst. Sie enthält Annahmen dazu, welche erwerbsbiografischen und sozio-demografischen Merkmale in den aus der bisherigen Empirie abgeleiteten Verlaufsmustern dominant sind sowie Zuordnungen der einzelnen atypischen Beschäftigungsformen zu den vermuteten Verläufen. Nach Darstellung unseres methodischen Vorgehens (3.) werden die in Tabelle 1 zusammengefassten Annahmen empirisch überprüft (4.) und im Anschluss daran diskutiert (5.).

Tabelle 1: Annahmen über Erwerbsverlaufsmuster unter Berücksichtigung atypischer Beschäftigung

\begin{tabular}{|c|c|c|c|}
\hline Erwerbsverlaufsmuster & $\begin{array}{l}\text { dominante erwerbs- } \\
\text { biografische und sozio- } \\
\text { demografische Merkmale }\end{array}$ & $\begin{array}{l}\text { vorrangige Formen der } \\
\text { atypischen Beschäftigung }\end{array}$ & Beispiele \\
\hline konstant atypisch & $\begin{array}{l}\text { Haupterwerbsphase } \\
\text { weiblich } \\
\text { Paarhaushalt mit Kindern } \\
\text { niedrige Qualifikation }\end{array}$ & $\begin{array}{l}\text { Teilzeit } \\
\text { geringfügige Beschäftigung }\end{array}$ & Vereinbarkeitsmodus \\
\hline episodenhaft atypisch & $\begin{array}{l}\text { Einstiegsphase } \\
\text { weiblich } \\
\text { niedrige Qualifikation }\end{array}$ & $\begin{array}{l}\text { geringfügige Beschäftigung } \\
\text { Leiharbeit }\end{array}$ & $\begin{array}{l}\text { Einmünden in den Arbeits- } \\
\text { markt, Pendeln zwischen } \\
\text { Arbeitslosigkeit und } \\
\text { Erwerbstätigkeit }\end{array}$ \\
\hline atypisch $\rightarrow$ regulär & $\begin{array}{l}\text { Einstiegsphase } \\
\text { männlich } \\
\text { hohe Qualifikation }\end{array}$ & Befristung & verlängerte Probezeit \\
\hline atypisch $\rightarrow$ arbeitslos & niedrige Qualifikation & $\begin{array}{l}\text { geringfügige Beschäftigung } \\
\text { Leiharbeit }\end{array}$ & $\begin{array}{l}\text { fehlende Qualifikation in } \\
\text { spezifischen Arbeitsmarkt- } \\
\text { segmenten }\end{array}$ \\
\hline atypisch $\rightarrow$ nichterwerbstätig & $\begin{array}{l}\text { Einstiegsphase } \\
\text { Endphase } \\
\text { weiblich } \\
\text { Paarhaushalt mit Kindern }\end{array}$ & $\begin{array}{l}\text { Teilzeit } \\
\text { geringfügige Beschäftigung }\end{array}$ & Elternschaft, Rente \\
\hline
\end{tabular}




\section{Methoden und Daten}

\subsection{Methoden}

Um der Frage nachzugehen, in welchen Verläufen atypische Beschäftigung in welcher Weise auftritt, führen wir eine Sequenzmuster- mit anschließender Clusteranalyse durch. Die Sequenzmusteranalyse ermöglicht eine holistische Betrachtung von Daten; aus der Empirie heraus werden Typologien von Sequenzen (hier: Erwerbsverläufe) identifiziert (Aisenbrey \& Fasang 2010). Zur Berechnung der Distanz zwischen zwei Sequenzen wird das Optimal Matching Verfahren angewandt (Abbott \& Tsay 2000). ${ }^{4}$

Der Optimal-Matching-Algorithmus erstellt noch keine Typen oder Klassifikationen, sondern berechnet die Anzahl der minimal nötigen Transformationen, um eine Sequenz in eine andere zu verwandeln. Dabei wird nicht nur die Länge und Häufigkeit von Zuständen (hier: verschiedene Erwerbsstatus) berücksichtigt, sondern auch deren Position im Verlauf und ihre Reihenfolge. ${ }^{5}$

An die Optimal Matching Analyse schließt eine Clusteranalyse zur Klassifikation bzw. Typenbildung der Erwerbsverläufe entlang der Empirie an. Ziel ist die Bildung möglichst homogener Cluster, die sich deutlich voneinander unterscheiden. Die jeweiligen Regeln beeinflussen dabei die Lösungen für die Clusterbildung maßgeblich. In der Praxis wird derzeit bevorzugt auf das Ward-Verfahren zurückgegriffen (Aisenbrey \& Fasang 2010; Klenner \& Schmidt 2012; Kogan 2003; Scherer 2001), da es vergleichsweise gute Ergebnisse, d.h. homogene Cluster erzeugt. Dabei muss allerdings zunächst über die „optimale“ Anzahl der Cluster entschieden werden. Da die Verwendung der gängigen Teststatistiken im Falle einer vorangegangen Sequenzmusteranalyse nicht möglich ist (Aisenbrey \& Fasang

\footnotetext{
${ }^{4}$ In unserer Analyse verwenden wir für die Durchführung von Optimal-Matching sowie für die spätere Darstellung der Sequenzmuster die von Brzinsky-Fay et al. (2006) programmierten SQ-Ados für Stata.

${ }_{5}^{5}$ Um diesen Anforderungen Rechnung zu tragen, ist zunächst eine Spezifikation von Kosten zur Substitution verschiedener Zustände nötig. In Ermangelung einer theoretisch begründbaren Kostenstruktur setzen wir die Kosten zur Substitution eines Erwerbsstatus durch einen anderen jeweils auf eins. Des Weiteren setzen wir die Kosten zum Löschen/Einsetzen von Zuständen auf 0,5. Wie bisherige Anwendungen zeigen (Scherer \& Brüderl 2010), erweisen sich auch die Ergebnisse der vorliegenden Analyse gegenüber einer Veränderung der Transformationskosten als robust.
}

2010), entscheiden wir über die Anzahl der Cluster mithilfe von Dendrogrammen sowie nach der Interpretierbarkeit der Clusterlösung auf der Grundlage von Sequenzindexplots.

\subsection{Datensatz und Operationalisierungen}

Datengrundlage der empirischen Analyse ist die vom Deutschen Institut für Wirtschaftsforschung (DIW) bereitgestellte Längsschnittstudie Soziooekonomisches Panel (SOEP). Das SOEP ist eine repräsentative Wiederholungsbefragung privater Haushalte in Deutschland, die seit 1984 im jährlichen Rhythmus bei denselben Personen und Haushalten erhoben wird (Wagner et al. 2007). Unter Einbezug der Erhebungswellen von 2002 bis 2011 erstellen wir einen balancierten Datensatz auf der Grundlage von jährlichen Informationen. ${ }^{6}$ Es werden alle Nichterwerbspersonen sowie Erwerbspersonen zwischen 16 und 69 Jahren in den Datensatz aufgenommen. Wir führen die Sequenzmuster- und Clusteranalysen getrennt für drei Geburtskohorten (Geburtsjahrgänge von 1944 bis 1952, 1957 bis 1970 sowie 1977 bis 1985) durch. In unserem Beobachtungszeitraum von 2002 bis 2011 befindet sich die jüngste Kohorte in ihrer Erwerbseinstiegsphase, die beiden anderen Kohorten in der Haupterwerbs- bzw. in der Erwerbsendphase. ${ }^{7}$ Wünschenswert wäre eine Beobachtung verschiedener Geburtskohorten in den jeweils drei Phasen ihrer Erwerbsbiografie, um Alters-, Kohorten- und Erwerbsphaseneffekte eindeutig identifizieren zu können. Hierfür wäre aber die Verwendung mehrerer Beobachtungszeiträume nötig. Unsere Datengrundlage lässt dies jedoch nicht $\mathrm{zu}$, da sich atypische Beschäftigungsverhältnisse ausschließlich in dem gewählten Beobachtungszeitraum voneinander abgrenzen lassen.

${ }^{6}$ Gegen die Verwendung von monatlichen Daten des SOEP spricht, dass damit nicht alle atypischen Erwerbsformen voneinander abgegrenzt werden können. Zur Konstruktion des Datensatzes haben wir das Programm PanelWhiz (Haisken-DeNew \& Hahn 2010) verwendet.

7 Die Geburtskohorten wurden so gewählt, dass sie sich über ihr Alter während des Beobachtungszeitraums jeweils einer Erwerbsphase eindeutig zuordnen lassen. Für unseren Beobachtungszeitraum 2002-2011 ergibt sich somit folgende Altersstruktur der Personen in den drei Erwerbsphasen: Personen in der Einstiegsphase sind zu Beginn des Beobachtungszeitraums zwischen 17 und 25 Jahre alt und zwischen 26 und 34 Jahren zu dessen Ende. Personen in der Hauptphase: 32-45 Jahre zu Beginn und 41-54 Jahre zum Ende. Personen in der Endphase: 50-58 Jahre zu Beginn und 59-67 Jahre zum Ende. 
Tabelle 2: Operationalisierung der Variable Erwerbsstatus und der in Abschnitt 4.3 verwendeten sozio-demografischenVariablen

\begin{tabular}{ll}
\hline Erwerbsstatus, nominal & \\
\hline 1. Nichterwerbsperson & - nicht erwerbstätig und nicht arbeitslos gemeldet (z. B. Rente), Wehr-/Zivildienst \\
2. arbeitslos & - nicht erwerbstätig und beim Arbeitsamt gemeldet \\
3. atypisch & - Teilzeit, geringfügig/unregelmäßig beschäftigt, Mini-/Midi-Job, Leiharbeit oder befristet \\
4. regulär beschäftigt & - unbefristeter Vertrag in Vollzeit, keine Leiharbeit \\
5. selbstständig & - selbstständig \\
6. Ausbildung / Schule & - in Ausbildung / Schule / Studium (exklusive Umschulung und Weiterbildung)
\end{tabular}

\section{Sozio-demografische Merkmale}

Bildung, Dummy

in Schule

niedrig kein Abschluss, Hauptschulabschluss ohne berufliche Ausbildung

mittel Hauptschulabschluss und berufliche Ausbildung, Mittlere Reife ohne berufliche Ausbildung, Mittlere Reife und berufliche Ausbildung, Fachhochschulreife/Abitur ohne berufliche Ausbildung, Fachhochschulreife/Abitur und berufliche Ausbildung

hoch Fachhochschulabschluss, Hochschulabschluss

Haushaltstyp, Dummy

Alleinlebend

Einpersonenhaushalt ohne Kinder

Paar ohne Kinder

Paarhaushalt ohne Kinder

Alleinerziehend

Alleinerziehend mit verschiedener Kinderanzahl

Paar mit Kindern

Paarhaushalt mit verschiedener Kinderanzahl

andere

3- und 4-Generationen-Haushalt, Großeltern-Kind(er)-Haushalt, andere

Bemerkung: Jeder Person kann nur ein Erwerbsstatus pro Zeitpunkt zugeordnet werden. Wenn für eine Person pro Zeitpunkt mehrere Status zutreffen, wird ihr jeweils der Status mit der höheren Nummerierung zugeschrieben. Beispiel: Ist eine Person in einem Minijob beschäftigt, studiert jedoch zugleich, wird sie der Kategorie „Ausbildung / Studium“ zugeordnet. Personen, die eine Altersrente, Pension, oder Invalidenrente beziehen und gleichzeitig einer Erwerbstätigkeit nachgehen, werden als Nichterwerbspersonen behandelt. Die Einordnung in Voll- oder Teilzeitbeschäftigung beruht auf einer Selbsteinschätzung der Befragten. Personen in Arbeitsbeschaffungsmaßnahmen sowie Ein-Euro-Jobs wurden aus der Analyse ausgeschlossen.

Die detaillierte Operationalisierung des Erwerbsstatus, die Grundlage der Sequenzmuster- und Clusteranalysen ist, findet sich in Tabelle 2. Die einzelnen Formen atypischer Beschäftigung werden zunächst zusammengefasst in einer Kategorie betrachtet. Erst an späterer Stelle nehmen wir eine Differenzierung der atypischen Erwerbsformen vor, wobei wir uns ausschließlich auf abhängig Beschäftigte konzentrieren. Weil mehrere Merkmale atypischer Beschäftigung auf ein Beschäftigungsverhältnis zutreffen können, haben wir zur Unterscheidung einzelner atypischer Beschäftigungsformen folgende Hierarchie verwendet: Personen, die angegeben, sich in einem Leiharbeitsverhältnis zu befinden, werden diesem zugeordnet, auch wenn auf ihr Beschäftigungsverhältnis noch andere Merkmale atypischer Beschäftigung zutreffen wie z.B. Teilzeit oder Befristung. Des Weiteren werden auch Mini- oder
Midijobs, ${ }^{8}$ auf die das Merkmal Befristung zutrifft, als solche betrachtet und nicht etwa als befristete Beschäftigungen. In Tabelle 2 finden sich zudem die Operationalisierungen der sozio-demografischen Merkmale für die weitere Analyse.

Die Sequenzindexplots (Grafiken A1, A2, und A3 im Online-Anhang) zeigen für jede der drei Erwerbsphasen alle Erwerbsverläufe der Untersuchungsstichprobe. Jeder einzelne Verlauf wird als eine horizontale Line dargestellt und gemäß des oben beschriebenen Algorithmus einem Cluster zugeordnet. Die sechs möglichen Erwerbsstatus, die $\mathrm{zu}$ den jeweiligen auf der $\mathrm{x}$-Achse abgetragenen Zeitpunkten angenommen werden können, sind

${ }^{8}$ Da eine Unterscheidung zwischen Mini- und Midijobs mit Daten des SOEP erst ab dem Jahr 2004 möglich ist, behandeln wir sie im Rahmen unserer Analysen gemeinsam. 
farbig dargestellt. Für die Untersuchung von Erwerbsverläufen sind Sequenzindexplots von besonderer Bedeutung, weil sie die zeitliche Abfolge der Erwerbsstatus sichtbar machen. Darüber hinaus untersuchen wir die Verläufe mithilfe von statistischen Maßzahlen, die wir in Tabelle A1 (OnlineAnhang) darstellen. ${ }^{9}$

\section{Empirische Analyse}

Um unsere Frage zu beantworten, in welche Verlaufstypen atypische Beschäftigung in welcher Weise eingebettet ist, nehmen wir zunächst eine Bezeichnung aller vorliegenden Cluster nach dem hauptsächlich vorliegenden Erwerbstatus vor (Tabelle 3). Vorliegende Wechsel zwischen Erwerbsstatus sind mit einem Pfeil markiert. Die Tabelle gibt auch Auskunft über die Größe des Clusters, d.h. über die Anzahl der im Cluster enthaltenen Personen. Um einen Eindruck von den Größenverhältnissen zu erhalten, ist außerdem der Anteil aller untersuchten Personen einer Erwerbsphase angegeben, die sich in den Clustern befinden. Dieser Anteilswert wurde gewichtet, um eine Annäherung an die tatsächliche Größenverteilung von Erwerbsverlaufsmustern in der Bevölkerung zu erreichen. ${ }^{10}$

Am augenfälligsten ist, dass Cluster, die eine konstante reguläre Beschäftigung über den gesamten Beobachtungszeitraum zusammenfassen, größenmäßig dominieren. In der Haupterwerbsphase sind 40,4 \% der Personen einem solchen Cluster zuzuordnen. In der Erwerbseinstiegs- und Endphase ist dieser Anteil geringer (15,4 bzw. 32,5\%), was u. a. der Existenz von Clustern geschuldet ist, die Einstiege und Ausstiege aus dem Arbeitsmarkt be-

\footnotetext{
${ }^{9}$ Wir geben dort für jedes Cluster die durchschnittliche Anzahl der Beobachtungszeitpunkte an, zu denen ein bestimmter Erwerbsstatus vorliegt. Zusätzlich geben wir für jedes Cluster die Verteilung der Anzahl von Episoden an, die durch das ununterbrochene Auftreten atypischer Beschäftigung gekennzeichnet sind.

${ }^{10}$ Dabei wurde ein Längsschnittgewicht konstruiert, das unterschiedliche Auswahlwahrscheinlichkeiten sowie unterschiedliche Ausfallwahrscheinlichkeiten im Panel korrigieren soll. Bei der weiteren Analyse der Cluster bzw. Verlaufsformen verzichten wir auf eine solche Gewichtung. Verallgemeinerungen über die Untersuchungsstichprobe hinaus halten wir jedoch auch hier für zulässig, da wir Schlüsse hauptsächlich aus Vergleichen der Größenverhältnisse von Merkmalsausprägungen der untersuchten Variablen zwischen den Clustern bzw. Verlaufsformen ziehen. Die Höhe dieser Anteils- bzw. Durchschnittswerte kann dabei von den tatsächlichen Werten innerhalb der Bevölkerung abweichen.
}

obachten und somit das Muster einer konstant regulären Beschäftigung zwangsläufig durchbrechen. Nichtsdestotrotz bestätigen diese Ergebnisse die vielerorts formulierte Annahme einer nach wie vor höchst bedeutsamen Rolle der von durchgängig regulärer Beschäftigung geprägten Normalarbeitsbiografie.

\subsection{Muster atypischer Erwerbsverläufe}

Im Folgenden nehmen wir unserer Fragestellung gemäß solche Erwerbsverläufe in den Blick, die von der Normalarbeitsbiografie abweichen. Die entsprechenden Cluster haben wir in Tabelle 3 grau hinterlegt.

Konstant atypische Erwerbsverläufe spielen anteilsmäßig in der Haupterwerbsphase die größte Rolle. Gemäß unserer Zuordnung (siehe Tabelle 3, letzte Spalte) machen sie dort $21 \%$ aller Verläufe aus. Atypische Beschäftigung wird in den entsprechenden Clustern im Durchschnitt zu 9,5 (Cluster 5) bzw. 7 (Cluster 4) von 10 Zeitpunkten ausgeübt. In den beiden anderen Erwerbsphasen spielen konstant atypische Verläufe eine deutlich geringere Rolle. In der Endphase charakterisieren wir 6,4\% der Verläufe als konstant (Cluster 7; 9,3 Zeitpunkte im Durchschnitt), in der Einstiegsphase sind es nur $1,4 \%$ der Verläufe (Cluster 2; 7,8 Zeitpunkte). Diese Ergebnisse entsprechen unseren theoretischen Überlegungen (Tabelle 1): Atypische Beschäftigung tritt in der Haupterwerbsphase stärker in konstanter Form auf als in den beiden anderen Erwerbsphasen.

Grundsätzlich besteht jedoch die Gefahr, dass durch Links- bzw. Rechtszensierungen der analysierten Daten die Rolle von konstanter atypischer Beschäftigung in der Einstiegs- und Endphase unterschätzt wird. So stellt sich bspw. die Frage, ob Cluster in der Einstiegsphase (Cluster 4 und 8), die einen Übergang aus dem Bildungssystem in atypische Beschäftigung abbilden, nicht einen konstant atypischen Beschäftigungsverlauf zeigen würden, wenn man die Personen erst ab dem Zeitpunkt ihrer ersten Beschäftigung nach dem Verlassen des Bildungssystems beobachtet hätte. Für einen Großteil der atypischen Beschäftigungen, die direkt an eine Bildungsphase anschließen, ist allerdings ersichtlich, dass sie über den Beobachtungszeitraum hinweg einmal oder mehrfach unterbrochen wird, was sich in der hohen Anzahl der Episoden atypischer Beschäftigung zeigt (Cluster 4, 8, Einstiegsphase, Tabelle A1 im Online-Anhang). Diese Unterbrechungen sind unseres Erachtens so gewichtig, dass 
Tabelle 3: Erwerbsverlaufscluster in drei Erwerbsphasen: Bezeichnungen, Größenverhältnisse und Zuordnung zu Verlaufsmustern atypischer Beschäftigung

\begin{tabular}{|c|c|c|c|c|c|}
\hline Phase & Cluster & Bezeichnung des Clusters & $\mathbf{N}$ & \% gew. & $\begin{array}{l}\text { Verlaufsmuster atypischer } \\
\text { Beschäftigung }\end{array}$ \\
\hline \multirow{13}{*}{ 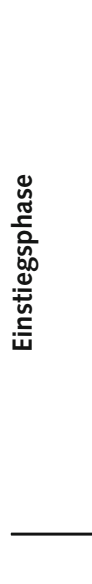 } & 1 & Nicht erwerbstätig & 72 & 8,3 & \\
\hline & 2 & Atypische Beschäftigung & 25 & 1,4 & konstant \\
\hline & 3 & Regulär $\rightarrow$ atypisch (episodenhaft) & 26 & 6,4 & episodenhaft \\
\hline & 4 & Ausbildung/Schule $\rightarrow$ atypisch & 50 & 6,6 & episodenhaft \\
\hline & 5 & Ausbildung/Schule $\rightarrow$ arbeitslos & 47 & 4,7 & \\
\hline & 6 & Ausbildung/Schule $\rightarrow$ selbstständig & 24 & 2,9 & \\
\hline & 7 & Ausbildung/Schule & 219 & 15,0 & \\
\hline & 8 & Ausbildung/Schule (lang) $\rightarrow$ atypisch/regulär & 63 & 7,5 & episodenhaft \\
\hline & 9 & Reguläre Beschäftigung & 136 & 15,4 & \\
\hline & 10 & Ausbildung/Schule (kurz) $\rightarrow$ regulär & 85 & 12,3 & \\
\hline & 11 & Atypisch $\rightarrow$ regulär & 42 & 4,5 & atypisch $\rightarrow$ regulär \\
\hline & 12 & Ausbildung/Schule $\rightarrow$ atypisch (kurz) $\rightarrow$ regulär & 110 & 15,2 & atypisch $\rightarrow$ regulär \\
\hline & & & 899 & 100 & \\
\hline \multirow{12}{*}{ 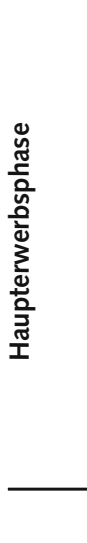 } & 1 & Nicht erwerbstätig & 248 & 8,9 & \\
\hline & 2 & Arbeitslos & 72 & 2,2 & \\
\hline & 3 & Arbeitslos/atypisch beschäftigt & 103 & 5,6 & episodenhaft \\
\hline & 4 & Nicht erwerbstätig (kurz) $\rightarrow$ konstant atypisch & 158 & 6,3 & konstant \\
\hline & 5 & Atypische Beschäftigung & 480 & 14,7 & konstant \\
\hline & 6 & Atypisch $\rightarrow$ regulär & 95 & 2,7 & atypisch $\rightarrow$ regulär \\
\hline & 7 & Selbstständig und andere Zustände & 159 & 4,5 & \\
\hline & 8 & Selbstständig & 218 & 5,6 & \\
\hline & 9 & Atypisch $\rightarrow$ regulär & 182 & 5,9 & atypisch $\rightarrow$ regulär \\
\hline & 10 & Reguläre Beschäftigung & 1392 & 40,4 & \\
\hline & 11 & Regulär/atypisch beschäftigt (punktuell) & 112 & 3,3 & \\
\hline & & & 3219 & 100 & \\
\hline \multirow{11}{*}{ 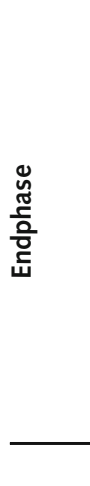 } & 1 & Nicht erwerbstätig & 319 & 22,7 & \\
\hline & 2 & Arbeitslos $\rightarrow$ nicht erwerbstätig & 79 & 4,2 & \\
\hline & 3 & Regulär $\rightarrow$ nicht erwerbstätig & 44 & 2,7 & \\
\hline & 4 & Regulär $\rightarrow$ nicht erwerbstätig & 123 & 6,1 & \\
\hline & 5 & Arbeitslos & 82 & 6,6 & \\
\hline & 6 & Regulär/atypisch/arbeitslos & 60 & 4,1 & episodenhaft \\
\hline & 7 & Atypische Beschäftigung & 126 & 6,4 & konstant \\
\hline & 8 & Atypisch $\rightarrow$ nicht erwerbstätig & 98 & 7,8 & atypisch $\rightarrow$ Nichterwerb \\
\hline & 9 & Selbstständig & 136 & 7,0 & \\
\hline & 10 & Reguläre Beschäftigung & 540 & 32,5 & \\
\hline & & & 1607 & 100 & \\
\hline
\end{tabular}

Daten: SOEP 2002-2011, eigene Berechnungen. Die Nummerierung der Cluster entspricht derjenigen der Sequenzindexplots (Grafiken $A 1, A 2$, und $A 3)$ im Online-Anhang. 
sie die Charakterisierung als episodenhafte Verläufe rechtfertigen. Auch in der Endphase beobachten wir Phasen atypischer Beschäftigung von hoher Konstanz (Cluster 8). Weil im Rahmen dieser Verläufe jedoch ein Übergang in die Nichterwerbstätigkeit stattfindet, diskutieren wir sie weiter unten als ein diesem Übergang entsprechendes Muster.

Unseren Erwartungen entsprechend sind episodenhaft atypische Verläufe, d.h. Erwerbsverläufe, in denen sich atypische Beschäftigungsepisoden mit anderen Erwerbsstatus abwechseln, insbesondere in der Erwerbseinstiegsphase stark vertreten. Wir charakterisieren 20,5\% aller Verläufe in dieser Phase als episodenhaft atypisch (Cluster 3, 4 und 8, Einstiegsphase). Atypische Beschäftigung folgt hier bspw. auf eine kurze Nichterwerbsperiode, der reguläre Beschäftigung vorausging (Cluster 3, Einstiegsphase). Separat durchgeführte Analysen zeigen, dass es sich dabei in den meisten Fällen um Wiedereinsteigerinnen in Beschäftigung nach der Geburt eines Kindes handelt. Atypische Beschäftigung tritt auch gehäuft nach dem Übergang aus dem Bildungssystem auf und wechselt mit Phasen von Arbeitslosigkeit oder regulärer Beschäftigung (Cluster 4 und 8, Einstiegsphase). In der Haupterwerbsphase charakterisieren wir 5,6\% aller Verläufe als episodenhaft (Cluster 3, Hauptphase). Atypische Beschäftigungen finden dort hauptsächlich im Wechsel mit Arbeitslosigkeit statt. In der Erwerbsendphase gilt das für $4,1 \%$ der Verläufe (Cluster 6, Endphase), wobei sich atypische Beschäftigung dort zwischen Phasen der regulären Beschäftigung und/oder Arbeitslosigkeit schiebt.

Wechsel von atypischer in reguläre Beschäftigung lassen sich als Verlaufsmuster nur in der Einstiegsund Hauptphase des Erwerbslebens feststellen. Anteilsmäßig sind sie in der Einstiegsphase mit 19,7 \% (Cluster 11 und 12) deutlich stärker vertreten als in der Haupterwerbsphase (8,6\%, Cluster 6 und 9). Reguläre Beschäftigungen schließen sich in der Einstiegsphase sowohl an längere als auch an kurze Phasen atypischer Beschäftigung an. Der Wechsel findet in der Tendenz zur Mitte des Beobachtungszeitraums statt. In der Hauptphase sind atypische Beschäftigungsverhältnisse häufig Episoden, die in einen ansonsten regulären Verlauf eingebettet sind.

Wechsel von atypischer Beschäftigung in Arbeitslosigkeit liegen in keiner der Erwerbsphasen in Form eines Clusters vor. Es konnte keine Verlaufsform identifiziert werden, in der längere Phasen von Arbeitslosigkeit an eine Form von atypischer Beschäftigung anschließen. Stattdessen folgen Phasen von Arbeitslosigkeit in der Einstiegsphase di- rekt auf Schule und Ausbildung. In der Haupterwerbsphase stellen durch lang anhaltende Arbeitslosigkeit geprägte Verläufe ein eigenes Cluster dar, oder die Arbeitslosigkeit wird, wie oben beschriebenen, durch atypische Beschäftigungsepisoden unterbrochen. Ein Prozess des Ausschlusses aus dem Arbeitsmarkt über atypische Beschäftigung ist somit nicht nachzuvollziehen.

Wechsel von atypischer Beschäftigung in Nichterwerb konnten wir nur in der Erwerbsendphase als eigenständige Verlaufsform identifizieren. Es handelt sich dabei um Rentenübergänge (Cluster 8). Für mehr als $70 \%$ der Personen in diesem Cluster ist atypische Beschäftigung die einzige über einen längeren Zeitraum (im Durchschnitt fünf Jahre) ausgeübte Erwerbsform vor dem Renteneintritt. In weiteren Clustern sind kürzere Episoden atypischer Beschäftigung vor dem Renteneintritt erkennbar (2, 3, 4, Endphase), nicht selten eingebettet in einen ansonsten regulären Verlauf (Cluster 4, Endphase).

Weitere Verlaufsmuster atypischer Beschäftigung lassen sich erkennen, die in unseren theoretischen Überlegungen bislang keine Rolle spielten. So kommen atypische Beschäftigungen gehäuft in punktueller Form im Rahmen von ansonsten regulären Verläufen vor. $20 \%$ der konstant regulär beschäftigten Personen in der Haupterwerbsphase berichten mindestens eine Episode atypischer Beschäftigung. Im Folgenden behandeln wir diese Verläufe jedoch nicht als atypische Erwerbsverläufe.

Im Hinblick auf unsere Annahmen in Tabelle 1 lässt sich zusammenfassend sagen: Es bestätigt sich, dass konstant atypische Verläufe in der Haupterwerbsphase, episodenhaft atypische Verläufe und der Übergang von atypische in reguläre Beschäftigung in der Einstiegsphase dominieren, jeweils verglichen mit ihren Ausprägungen in den anderen Erwerbsphasen. Entgegen unseren Annahmen beschränken sich Verläufe, die durch einen Übergang von atypischer Beschäftigung in Nichterwerb gekennzeichnet sind, auf die Erwerbsendphase (Rentenübergänge). Ebenso lässt sich kein Muster erkennen, dass das Ausscheiden aus dem Arbeitsmarkt in Form von Arbeitslosigkeit über atypische Beschäftigung moderierte. Stattdessen sehen wir in der Haupterwerbsphase die Tendenz, dass Arbeitslosigkeitsverläufe durch atypische Beschäftigungen unterbrochen werden. 


\subsection{Erwerbsverlaufsmuster und atypische Beschäftigungsformen}

Um Aufschluss darüber zu erhalten, welche atypischen Beschäftigungsformen die gefundenen Verlaufsmuster atypischer Beschäftigung spezifisch prägen, berechnen wir die Anteile der atypischen Beschäftigungsformen an allen atypischen Beschäftigungen pro Verlaufsmuster (Tabelle 4). Dabei zeigen sich deutliche Unterschiede in der Häufigkeit des Auftretens bestimmter atypischer Beschäftigungsformen zwischen den atypischen Verlaufsmustern sowie zwischen den drei Erwerbsphasen.

Konstant atypische Verläufe setzen sich in allen Erwerbsphasen hauptsächlich aus unbefristeten Teilzeitbeschäftigungen zusammen $(54,9 \%$ Einstiegsphase, 60,5\% Hauptphase, 69,3\% Endphase). Damit werden unsere Erwartungen (Tabelle 1) bestätigt. In der Haupt- bzw. Endphase stellen Miniund Midijobs (28,2 bzw. 20,1 \%) den zweitgrößten Anteil im Rahmen von konstant atypischen Verläufen. Leiharbeit und Befristungen spielen hingegen kaum eine Rolle. Konstant atypische Verläufe in der Einstiegsphase weichen jedoch von diesem Muster ab. Mini- und Midijobs sind dort weniger stark vertreten als in der Haupt- und Endphase. Stattdessen machen befristete Beschäftigungen in der Einstiegsphase einen vergleichsweise hohen Anteil der atypischen Beschäftigungen in konstant aty- pischen Verläufen aus. Dieser Befund verweist auf mögliche Befristungsketten bei Erwerbseinsteigern, was jedoch durch die geringe Anzahl von konstant atypischen Verläufen in der Einstiegsphase relativiert wird.

Über alle Erwerbsphasen hinweg machen Miniund Midijobs auch in den episodenhaften Verläufen einen großen Anteil atypischer Beschäftigungsformen aus. In der Haupterwerbsphase stellen sie mit $34 \%$ den größten Anteil aller atypischen Erwerbsformen im Rahmen von episodenhaften Verläufen dar. Auch Leiharbeit ist hier stark vertreten, befristete Beschäftigung hingegen nicht. Die anderen beiden Erwerbsphasen weisen jeweils andere Muster auf. In der Einstiegsphase sind befristete Vollzeitbeschäftigungen mit $37 \%$ die häufigste atypische Beschäftigungsform in den episodenhaften Verläufen. Episodenhafte Verläufe in der Erwerbsendphase sind durch Mini- und Midijobs und unbefristete Teilzeitbeschäftigungen geprägt, Befristungen und Leiharbeit kommen kaum vor (s. oben, 2.2). Insgesamt ist das Auftreten von atypischen Beschäftigungsformen im Rahmen von episodenhaft atypischen Verläufen sehr phasenspezifisch und folgt bis auf das häufige Auftreten von Mini- und Midijobs keinem übergreifenden Muster.

Wechsel von atypischer in reguläre Beschäftigung finden in der Haupterwerbsphase entgegen unseren

Tabelle 4: Anteil einer atypischen Beschäftigungsform an allen atypischen Beschäftigungen pro Verlaufsmuster, Spaltenprozente

\begin{tabular}{|c|c|c|c|c|c|c|c|c|c|}
\hline & \multicolumn{3}{|c|}{ Einstiegsphase } & \multicolumn{3}{|c|}{ Haupterwerbsphase } & \multicolumn{3}{|c|}{ Endphase } \\
\hline & 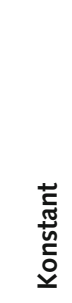 & $\begin{array}{l}\frac{ \pm}{\pi} \\
\frac{\pi}{0} \\
\frac{0}{0} \\
\frac{0}{0} \\
\frac{0}{2}\end{array}$ & 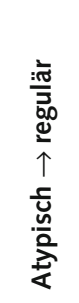 & 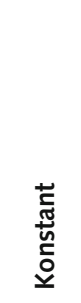 & 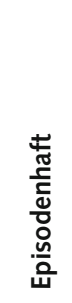 & 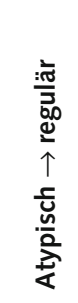 & 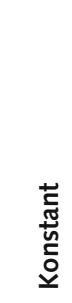 & 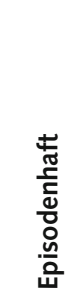 & 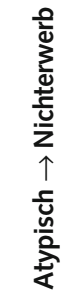 \\
\hline \multicolumn{10}{|l|}{ Atypische Erwerbsformen } \\
\hline Leiharbeit & 2,1 & 12,7 & 15,5 & 2,1 & 21,9 & 7,3 & 1,7 & 9,9 & 2,6 \\
\hline Mini/Midijob & 13,3 & 15,6 & 5,3 & 28,2 & 34,0 & 4,9 & 20,1 & 30,0 & 23,0 \\
\hline befristete Vollzeit & 14,4 & 37,0 & 52,9 & 1,8 & 12,4 & 16,9 & 1,9 & 9,9 & 1,2 \\
\hline befristete Teilzeit & 8,7 & 8,8 & 6,2 & 2,7 & 6,6 & 2,8 & 0,9 & 2,2 & 1,2 \\
\hline unbefristete Teilzeit & 54,9 & 18,5 & 13,6 & 60,5 & 14,7 & 51,5 & 69,3 & 34,2 & 60,6 \\
\hline \multirow{2}{*}{$\begin{array}{l}\text { sonstige, kein Arbeitsvertrag, } \\
\text { unregelmäßig beschäftigt }\end{array}$} & 6,7 & 7,4 & 6,5 & 4,6 & 10,4 & 16,8 & 6,2 & 13,7 & 11,4 \\
\hline & 100 & 100 & 100 & 100 & 100 & 100 & 100 & 100 & 100 \\
\hline
\end{tabular}

Daten: SOEP 2002-2011, eigene Berechnungen 
Erwartungen mehrheitlich aus unbefristeten Teilzeitbeschäftigungen heraus statt. Vermutlich handelt es sich dabei um Aufstockungen der Stundenanzahl in einem bestehenden Beschäftigungsverhältnis. In der Einstiegsphase sind es stattdessen vor allem befristete Vollzeitbeschäftigungen, die in Verbindung mit einem Wechsel in reguläre Beschäftigung stehen. Das unterstreicht die Bedeutung befristeter Beschäftigungsverhältnisse als Sprungbrett in reguläre Beschäftigung für Erwerbseinsteiger und legt die Vermutung nahe, dass die Befristung als eine Art Probezeit dient. In beiden Erwerbsphasen spielen Mini- und Midijobs bei Wechseln in reguläre Beschäftigung keine wesentliche Rolle. Sie gewähren zwar in der Haupterwerbsphase eine Beteiligung am Arbeitsmarkt, gehen jedoch dort und in der Erwerbseinstiegsphase nur selten mit Übergängen in reguläre Beschäftigung einher.

Wechsel von atypischer Beschäftigung in Nichterwerbstätigkeit, die wir nur in der Erwerbsendphase als Verlaufsmuster feststellen konnten, sind zu einem überwiegenden Anteil (60,6\%) durch unbefristete Teilzeitbeschäftigungen geprägt.

Zusammenfassend lässt sich sagen, dass die meisten unserer Annahmen über die Dominanz bestimmter atypischer Beschäftigungsformen in den einzelnen Verlaufstypen zutreffen, allerdings sehen wir eine starke Differenzierung nach Erwerbsphasen. Die konstant atypischen Verläufe bestehen mehrheitlich aus Teilzeitbeschäftigungen - ein Hinweis auf ihr vergleichsweise hohes Kontinuitätsversprechen. Auch Mini- und Midijobs spielen eine Rolle, aneinander gereihte Befristungen lediglich in der Einstiegsphase. Episodenhafte Verläufe bauen stark auf geringfügige Beschäftigung auf, was deren Charakter als kurzfristig einsetzbares Flexibilisierungsinstrument unterstreicht. Phasenspezifisch sehen wir aber darüber hinaus auch Episoden von Befristung (Eingangsphase), Leiharbeit (Hauptphase) und Teilzeit (Endphase). Befristungen lassen sich somit dem Forschungsstand und unseren Erwartungen entsprechend mehrheitlich in der beruflichen Einstiegsphase verorten, wo sie auch als Sprungbrett in reguläre Beschäftigung dienen können, geringfügige Beschäftigung hingegen ist phasenübergreifend sichtbar. Der Übergang in die Nichterwerbstätigkeit wird in Verläufen mit atypischer Beschäftigung in der beruflichen Endphase vornehmlich über Teilzeit bewerkstelligt.

\subsection{Erwerbsverlaufsmuster und sozio-demografische Merkmale}

Gender: Auch in Bezug auf sozio-demografische Merkmale sind die atypischen Verlaufsmuster stark strukturiert (Tabelle 5). Konstant atypische Verläufe werden in allen drei Erwerbsphasen zu einem extrem hohen Anteil von Frauen besetzt. In der Haupt- und Enderwerbsphase ist dies mit einem Frauenanteil von 95,9 bzw. 93,7\% nahezu ausschließlich der Fall. Der Übergang aus einer konstanten atypischen Beschäftigung in die Rente weist einen ähnlich hohen Anteil von Frauen auf. Alle sonstigen Verlaufsformen sind genderspezifisch weniger stark polarisiert. Auch in den episodenhaften Verläufen befinden sich zwar vermehrt Frauen. Wechsel von atypischer in reguläre Beschäftigung werden in der Einstiegsphase häufiger von Männern, in der Haupterwerbsphase entgegen unseren Annahmen häufiger von Frauen bewerkstelligt. Diese deutliche Genderspezifik atypischer Verlaufsmuster sollte jedoch nicht in Vergessenheit geraten lassen, dass sich Frauen wie Männer in allen Erwerbsphasen häufiger in anderen als atypischen Verläufen befinden (Zeilenprozente).

Bildung: Atypische Verläufe sind vergleichsweise häufig an ein geringes Bildungsniveau gekoppelt. Insbesondere gilt dies für zwei Verlaufsmuster: Verläufe mit atypischer Beschäftigung in episodenhafter Form weisen in der Hauptphase eine Verbindung mit niedriger Bildung auf $(13,4 \%)$, ebenso der Übergang in die Rente aus atypischer Beschäftigung in der Endphase (19,0\%). Im Vergleich dazu ist der Anteil der Personen mit geringer Bildung in den anderen atypischen Verlaufsmustern nur ungefähr halb so hoch. Konstant atypische Verläufe sind entgegen unserer Annahme nicht systematisch an ein geringes Bildungsniveau gekoppelt. Über alle Erwerbsphasen hinweg sind Personen mit einer niedrigen Bildung dort sogar vergleichsweise selten vertreten. Die Annahme, dass ein hohes Bildungsniveau bzw. hohe Qualifikationen ein dominantes Merkmal von Wechseln aus atypischer in reguläre Beschäftigung sind, kann hingegen sowohl für die Haupterwerbsphase und abgeschwächt auch für die Erwerbseinstiegsphase bestätigt werden. In beiden Erwerbsphasen stellen hohe Qualifikationen jedoch keine Garantie für Wechsel in reguläre Beschäftigung dar. Besonders gilt dies für die Haupterwerbsphase, wo diese Verlaufsform nur wenig verbreitet ist.

Haushaltsform: Gemäß unseren Annahmen sind Paarhaushalte mit Kindern die dominante Haushaltsform bei konstant atypisch Beschäftigten, ins- 
Tabelle 5: Verlaufsmuster atypischer Beschäftigung und sozio-demografische Merkmale in 2011, nach Erwerbsphase (Spalten- und Zeilenprozente)

\begin{tabular}{|c|c|c|c|c|c|c|c|c|c|c|c|c|c|}
\hline \multirow{2}{*}{\multicolumn{2}{|c|}{$\begin{array}{l} \\
\text { Sozio-demografische } \\
\text { Merkmale }\end{array}$}} & \multicolumn{4}{|c|}{ Einstiegsphase } & \multicolumn{4}{|c|}{ Haupterwerbsphase } & \multicolumn{4}{|c|}{ Endphase } \\
\hline & & 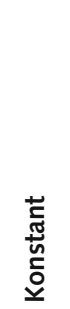 & $\begin{array}{l}\frac{\pi}{0} \\
\frac{\pi}{5} \\
\frac{0}{0} \\
\frac{0}{0} \\
\frac{n}{2}\end{array}$ & 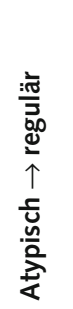 & $\begin{array}{l}\frac{0}{d} \\
\frac{\sigma}{\sigma}\end{array}$ & 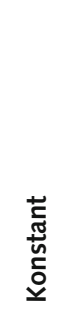 & $\begin{array}{l}\frac{\pi}{\pi} \\
\frac{\pi}{c} \\
\frac{0}{0} \\
\frac{0}{0} \\
\frac{n}{2}\end{array}$ & 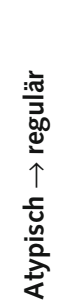 & 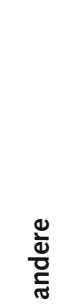 & 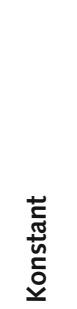 & \multicolumn{2}{|r|}{ 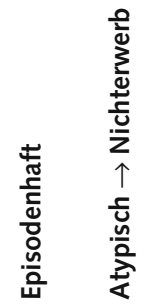 } & $\frac{\text { d̦ }}{\frac{d}{0}}$ \\
\hline Geschlecht & weiblich & $\begin{array}{r}88,0 \\
4,5\end{array}$ & $\begin{array}{l}61,9 \\
17,4\end{array}$ & $\begin{array}{l}44,1 \\
13,6\end{array}$ & $\begin{array}{l}54,6 \\
64,5\end{array}$ & $\begin{array}{l}95,9 \\
35,8\end{array}$ & $\begin{array}{r}54,4 \\
3,3\end{array}$ & $\begin{array}{r}56,0 \\
9,1\end{array}$ & $\begin{array}{l}40,3 \\
51,9\end{array}$ & $\begin{array}{l}93,7 \\
13,9\end{array}$ & $\begin{array}{r}65,0 \\
4,6\end{array}$ & $\begin{array}{l}95,9 \\
11,0\end{array}$ & $\begin{array}{l}45,4 \\
70,5\end{array}$ \\
\hline & männlich & $\begin{array}{r}12,0 \\
0,7\end{array}$ & $\begin{array}{l}38,1 \\
13,1\end{array}$ & $\begin{array}{l}55,9 \\
20,9\end{array}$ & $\begin{array}{l}45,5 \\
65,3\end{array}$ & $\begin{array}{l}4,1 \\
1,7\end{array}$ & $\begin{array}{r}45,6 \\
3,1\end{array}$ & $\begin{array}{r}44,0 \\
8,1\end{array}$ & $\begin{array}{l}59,7 \\
87,1\end{array}$ & $\begin{array}{l}6,4 \\
1,1\end{array}$ & $\begin{array}{r}35,0 \\
2,8\end{array}$ & $\begin{array}{l}4,1 \\
0,5\end{array}$ & $\begin{array}{l}54,6 \\
95,6\end{array}$ \\
\hline \multirow[t]{3}{*}{$\begin{array}{l}\text { Bildung in } \\
2011\end{array}$} & niedrig & $\begin{array}{l}4,0 \\
2,6\end{array}$ & $\begin{array}{r}7,1 \\
23,7\end{array}$ & $\begin{array}{r}5,6 \\
21,1\end{array}$ & $\begin{array}{r}3,7 \\
52,6\end{array}$ & $\begin{array}{r}6,3 \\
21,1\end{array}$ & $\begin{array}{r}13,4 \\
6,8\end{array}$ & $\begin{array}{r}7,7 \\
11,1\end{array}$ & $\begin{array}{r}5,4 \\
61,1\end{array}$ & $\begin{array}{l}8,1 \\
7,1\end{array}$ & $\begin{array}{l}6,8 \\
2,9\end{array}$ & $\begin{array}{l}19,0 \\
12,9\end{array}$ & $\begin{array}{r}8,3 \\
77,1\end{array}$ \\
\hline & mittel & $\begin{array}{r}88,0 \\
4,1\end{array}$ & $\begin{array}{l}62,7 \\
14,6\end{array}$ & $\begin{array}{l}62,9 \\
16,7\end{array}$ & $\begin{array}{l}64,2 \\
64,6\end{array}$ & $\begin{array}{l}76,0 \\
22,1\end{array}$ & $\begin{array}{r}72,2 \\
3,2\end{array}$ & $\begin{array}{r}69,7 \\
8,7\end{array}$ & $\begin{array}{l}66,4 \\
66,0\end{array}$ & $\begin{array}{r}66,1 \\
8,1\end{array}$ & $\begin{array}{r}74,6 \\
4,4\end{array}$ & $\begin{array}{r}55,8 \\
5,2\end{array}$ & $\begin{array}{l}63,6 \\
82,3\end{array}$ \\
\hline & hoch & $\begin{array}{l}8,0 \\
0,8\end{array}$ & $\begin{array}{l}30,2 \\
14,6\end{array}$ & $\begin{array}{l}31,5 \\
17,3\end{array}$ & $\begin{array}{l}32,2 \\
67,3\end{array}$ & $\begin{array}{l}17,7 \\
14,0\end{array}$ & $\begin{array}{r}14,4 \\
1,8\end{array}$ & $\begin{array}{r}22,6 \\
7,7\end{array}$ & $\begin{array}{l}28,2 \\
76,5\end{array}$ & $\begin{array}{r}25,8 \\
7,3\end{array}$ & $\begin{array}{r}18,6 \\
2,5\end{array}$ & $\begin{array}{r}25,3 \\
5,5\end{array}$ & $\begin{array}{l}28,2 \\
84,6\end{array}$ \\
\hline \multirow[t]{5}{*}{$\begin{array}{l}\text { Haushalts- } \\
\text { form in } 2011\end{array}$} & alleinlebend & $\begin{array}{l}4,0 \\
0,6\end{array}$ & $\begin{array}{l}20,9 \\
17,2\end{array}$ & $\begin{array}{l}20,4 \\
18,3\end{array}$ & $\begin{array}{l}18,6 \\
63,9\end{array}$ & $\begin{array}{l}2,4 \\
4,3\end{array}$ & $\begin{array}{r}17,5 \\
5,1\end{array}$ & $\begin{array}{l}8,3 \\
6,5\end{array}$ & $\begin{array}{l}13,5 \\
84,1\end{array}$ & $\begin{array}{l}8,7 \\
4,4\end{array}$ & $\begin{array}{r}20,0 \\
4,7\end{array}$ & $\begin{array}{r}14,3 \\
5,5\end{array}$ & $\begin{array}{l}16,3 \\
85,4\end{array}$ \\
\hline & $\begin{array}{l}\text { Paar } \\
\text { ohne Kinder }\end{array}$ & $\begin{array}{r}20,0 \\
2,3\end{array}$ & $\begin{array}{l}18,0 \\
11,5\end{array}$ & $\begin{array}{l}29,6 \\
20,6\end{array}$ & $\begin{array}{l}24,6 \\
65,6\end{array}$ & $\begin{array}{l}17,2 \\
15,2\end{array}$ & $\begin{array}{r}17,5 \\
2,5\end{array}$ & $\begin{array}{r}23,5 \\
9,0\end{array}$ & $\begin{array}{l}24,1 \\
73,3\end{array}$ & $\begin{array}{r}66,7 \\
8,0\end{array}$ & $\begin{array}{r}61,7 \\
3,5\end{array}$ & $\begin{array}{r}70,4 \\
6,6\end{array}$ & $\begin{array}{l}65,0 \\
81,9\end{array}$ \\
\hline & $\begin{array}{l}\text { allein- } \\
\text { erziehend }\end{array}$ & $\begin{array}{r}12,0 \\
4,0\end{array}$ & $\begin{array}{r}9,4 \\
17,1\end{array}$ & $\begin{array}{l}4,0 \\
7,9\end{array}$ & $\begin{array}{r}9,3 \\
71,1\end{array}$ & $\begin{array}{r}9,1 \\
27,2\end{array}$ & $\begin{array}{l}25,2 \\
12,2\end{array}$ & $\begin{array}{r}9,8 \\
12,7\end{array}$ & $\begin{array}{r}4,6 \\
47,9\end{array}$ & $\begin{array}{r}2,4 \\
11,1\end{array}$ & $\begin{array}{r}6,7 \\
14,8\end{array}$ & $\begin{array}{l}0,0 \\
0,0\end{array}$ & $\begin{array}{r}1,5 \\
74,1\end{array}$ \\
\hline & $\begin{array}{l}\text { Paar mit } \\
\text { Kindern }\end{array}$ & $\begin{array}{r}64,0 \\
3,8\end{array}$ & $\begin{array}{l}49,6 \\
16,4\end{array}$ & $\begin{array}{l}44,7 \\
16,2\end{array}$ & $\begin{array}{l}45,9 \\
63,6\end{array}$ & $\begin{array}{l}70,4 \\
23,7\end{array}$ & $\begin{array}{r}39,8 \\
2,2\end{array}$ & $\begin{array}{r}57,8 \\
8,4\end{array}$ & $\begin{array}{l}56,8 \\
65,8\end{array}$ & $\begin{array}{l}21,4 \\
10,6\end{array}$ & $\begin{array}{r}10,0 \\
2,4\end{array}$ & $\begin{array}{r}14,3 \\
5,5\end{array}$ & $\begin{array}{l}15,7 \\
82,0\end{array}$ \\
\hline & andere & $\begin{array}{l}0,0 \\
0,0\end{array}$ & $\begin{array}{r}2,2 \\
20,0\end{array}$ & $\begin{array}{r}1,3 \\
13,3\end{array}$ & $\begin{array}{r}1,7 \\
66,7\end{array}$ & $\begin{array}{r}0,9 \\
20,0\end{array}$ & $\begin{array}{l}0,0 \\
0,0\end{array}$ & $\begin{array}{l}0,7 \\
6,7\end{array}$ & $\begin{array}{r}1,0 \\
73,3\end{array}$ & $\begin{array}{l}0,8 \\
4,4\end{array}$ & $\begin{array}{l}1,7 \\
4,4\end{array}$ & $\begin{array}{l}1,0 \\
4,4\end{array}$ & $\begin{array}{r}1,5 \\
87,0\end{array}$ \\
\hline
\end{tabular}

Daten: SOEP 2002-2011, eigene Berechnungen

besondere in der Haupterwerbsphase. ${ }^{11}$ Am Ende der Erwerbsbiografie konzentrieren sich Paare ohne Kinder im Haushalt in diesem Verlauf. Alleinlebende stellen in der Haupt- und Enderwerbsphase einen hohen Anteil der Personen in episodenhaft atypischen Verläufen. Grundsätzlich muss auch an dieser Stelle darauf hingewiesen werden, dass sich jede Haushaltsform häufiger in nicht atypischen Verläufen wiederfinden lässt. Eine Ausnahme stellen allerdings alleinerziehende Personen in der Haupterwerbsphase dar, die häufiger in atypischen als in anderen Verlaufsformen vertreten sind.

\footnotetext{
${ }^{11}$ In der Erwerbseinstiegsphase, in der konstant atypische Verläufe nur in sehr geringem Umfang vorliegen, beobachten wir ebenfalls einen hohen Anteil an Paarhaushalten mit Kindern. Darunter befinden sich jedoch auch atypisch beschäftigte Erwerbseinsteiger, die noch im Haushalt ihrer Eltern wohnen.
}

Zusammengefasst finden wir in konstant atypischen Verläufen größtenteils Frauen vor, die mit einem Partner und Kindern in einem gemeinsamen Haushalt leben. Wechsel von atypischer in reguläre Beschäftigung sind an mittlere und hohe Bildung gekoppelt. Eine Kumulation von Benachteiligungen deutet sich insbesondere bei Personen in episodenhaft atypischen Verläufen an, die häufig allein leben und niedrig gebildet sind.

\section{Diskussion}

Atypische Beschäftigung befindet sich mehrheitlich in klar abgrenzbaren Verlaufsmustern, deren Prävalenz erwerbsphasenspezifisch sowie nach Qualifikation, Geschlecht und Haushaltskontext variiert. Den Ergebnissen der Übergangsforschung entspre- 
chend haben sich sowohl konstant als auch episodenhaft atypische Verläufe ermitteln lassen, aber auch Verläufe, die sich durch Wechsel von atypischer in reguläre Beschäftigung oder in Nichterwerb auszeichnen. Hingegen haben wir keine Verläufe gefunden, die atypische Beschäftigung als Übergang in die längerfristige Arbeitslosigkeit ausweisen.

In episodenhaften Verläufen finden sich zwar Wechsel von atypischer Beschäftigung in Arbeitslosigkeit, aber auch wieder zurück. Dies verweist auf die geringe Distanz zwischen bestimmten Formen atypischer Beschäftigung, insbesondere Mini- und Midijobs, und Arbeitslosigkeit. Geringfügige Beschäftigung ist leicht $\mathrm{zu}$ verlieren, aber auch wieder aufzunehmen, was ihrem geringen sozialversicherungsrechtlichen Schutz sowie den flexiblen Einsatzmöglichkeiten und ihrer schnellen Kündbarkeit geschuldet ist. Insbesondere in der Haupterwerbsphase stehen diese episodenhaften Verläufe außerhalb oder am Rand des Arbeitsmarktes. Sie sind vergleichsweise häufig mit geringer oder fehlender Qualifikation verbunden. Weiterführende Analy$\operatorname{sen}^{12}$ haben gezeigt, dass die Nähe zu Einkommensarmut hier besonders groß ist und Alleinerziehende überrepräsentiert sind, was auf Erwerbsnotwendigkeiten in Kombination mit Restriktionen bei der Kinderbetreuung hindeutet. Atypische Beschäftigung ist in dieser Form und in diesem Verlauf nicht dazu geeignet, die materielle Existenz zu sichern und eine berufliche Aufstiegsperspektive zu gewähren.

Ein weiterer herausstechender Befund ist, dass sich eindeutige Abstiegsbewegungen, die von regulärer in atypische Beschäftigung führen würden, nicht als Muster erkennen lassen. Wer sich in regulärer Beschäftigung befindet, läuft wenig Gefahr, sich in diesem Sinne dauerhaft zu verschlechtern. Die Analyse zeigt die dominant vorherrschende und durch hohe Stabilität gekennzeichnete Einbindung in reguläre Beschäftigung über alle Erwerbsphasen hinweg. Atypische Beschäftigung tritt mehrheitlich in Erwerbsverläufen auf, die kaum Berührungspunkte mit dem Normalarbeitsverhältnis aufweisen.

Verläufe, die atypische Beschäftigung in spezifischer Weise integrieren, lassen sich am häufigsten in der Erwerbseinstiegsphase ausmachen. Hier sind

\footnotetext{
12 Wir haben die Verlaufsformen danach untersucht, inwieweit Personen im letzten Beobachtungsjahr, d. h. 2011, einen Mangel an finanziellen Ressourcen aufweisen. Als armutsgefährdet gelten alle Personen, die über weniger als $60 \%$ des mittleren Nettoäquivalenzeinkommens verfügen.
}

sie nicht nur zahlenmäßig stark vertreten, sondern auch in ihrem Auftreten heterogen. Stärker als in anderen Erwerbsphasen ist atypische Beschäftigung in Form von Befristungen dem Übergang in reguläre Beschäftigung vorgeschaltet. Dies gilt insbesondere für Männer. Niedrige Bildung erschwert diese Aufstiegsbewegung. In Ergänzung zum bisherigen Forschungsstand ist der Übergang von Befristung in reguläre Beschäftigung aber auch nicht auf Hochqualifizierte beschränkt. In der Einstiegsphase sehen wir darüber hinaus, wenn auch in geringem Umfang, bereits konstant atypische Verläufe, die sowohl aus Folgen befristeter Arbeitsverhältnisse als auch aus Teilzeitbeschäftigung bestehen. Befristungen stehen demnach durchaus ambivalent sowohl für einen Brücken- als auch für einen Klebeeffekt, ein Ergebnis, das den eher positiven Blick der Übergangsforschung auf Befristungen etwas trübt.

In der Haupterwerbsphase tritt atypische Beschäftigung verstärkt in kontinuierlicher Form auf und wird von Frauen in unbefristeter Teilzeit oder in Mini- und Midijobs dominiert. Es handelt sich mehrheitlich um Frauen in Paarhaushalten, häufig mit Kindern, mehrheitlich ohne Armutsrisiko. Unsere Analysen bestätigen damit den Befund, dass verfestigte atypische Beschäftigung weiblich und eine Folge genderspezifisch zugewiesener Verantwortlichkeiten für die Vereinbarkeit von Familie und Beruf ist. Mitunter kann sie in reguläre Beschäftigung überführt werden, dies gelingt Frauen in der Haupterwerbsphase besser als Männern, die oftmals schon in der beruflichen Einstiegsphase diesen Wechsel vollzogen haben.

Beim Erwerbsausstieg spielt atypische Beschäftigung eine verhältnismäßig geringe Rolle. Sie leitet mehrheitlich für Frauen als Teilzeitbeschäftigung und seltener als geringfügige Beschäftigung in die Rente über. Es lässt sich vermuten, dass dies Fortführungen konstant atypischer Verläufe aus der Haupterwerbsphase sind. Es handelt sich erneut vielfach um Paarhaushalte mit oder (schon) ohne Kinder(n). Auffällig ist das niedrige Bildungsniveau in diesen Verläufen.

Die Verbreitung bestimmter Verlaufstypen mit atypischer Beschäftigung ist somit nicht losgelöst von sozio-demografischen Merkmalen. Dies unterstreicht Spaltungstendenzen am Arbeitsmarkt und zeigt, dass atypische Beschäftigung für die breite Masse der Erwerbstätigen keine Realität ist. 


\section{Fazit}

Ziel dieses Artikels war es, die erwerbsverlaufsspezifische Dynamik atypischer Beschäftigung sichtbar zu machen und Einsichten in ihre sozialstrukturelle Kontextabhängigkeit zu gewinnen. Eine Destandardisierung von Erwerbsbiografien und die damit suggerierte schichtübergreifende Betroffenheit von atypischer Beschäftigung lassen sich tendenziell nur für die Erwerbseinstiegsphase nachweisen. Der große Block der konstanten atypischen Erwerbsverläufe von Frauen in der Haupterwerbsphase sowie deren Dominanz in unbefristeter Teilzeitbeschäftigung und Mini- bzw. Midijobs sind eher Ausdruck von verfestigten genderspezifischen Trennungslinien. Im aktuellen Haushaltskontext stehen diese Verläufe vermutlich nur selten mit materiellen Existenzsorgen in Verbindung und die mehrheitlich ununterbrochene Beschäftigung in Teilzeit signalisiert ein gewisses $\mathrm{Maß}$ an Stabilität. Im Hinblick auf individuelle soziale Sicherung jenseits des Haushalts, bspw. nach einer Scheidung, sind sie jedoch als prekär zu interpretieren. Atypische Beschäftigung hingegen, die sich mit Arbeitslosigkeit und Nichterwerb abwechselt, ist weder mit monetärer Sicherheit noch mit beruflichen Aufstiegsmöglichkeiten verbunden.

Sind die beschriebenen Strukturierungen aber als soziale Spaltungslinien zu verstehen, die auf die Polarisierung von Erwerbs- und Lebenschancen verweisen? Mit Blick auf die Tatsache, dass Verläufe mit atypischer Beschäftigung insgesamt einem dominanten und männlichen Block kontinuierlicher Vollzeitbeschäftigung gegenüberstehen und der Wechsel von atypischer in reguläre Beschäftigung vornehmlich Personen am Beginn ihres Erwerbslebens gelingt, trifft das durchaus zu. Die bei Frauen dominante Verlaufsform der konstanten Teilzeitbeschäftigung verweist aber nicht auf qualifikationsabhängige soziale Ungleichheit, sondern auf familienpolitisch zu verortende Vereinbarkeitsanstrengungen. Treffen niedrige Qualifikation, Phasen von Nichterwerb und Arbeitslosigkeit sowie Alleinelternschaft oder Singlehaushalt allerdings zusammen, so wird atypische Beschäftigung zum Prekaritätsrisiko. Insofern konzentriert sich atypische Beschäftigung in ihren jeweiligen Formen durchaus in bestimmten Erwerbsverläufen, die mit spezifischen sozio-demografischen Merkmalen in Verbindung stehen - und für eine kleine Gruppe von Beschäftigten erweisen sich die Erwerbs- und Lebenschancen als gefährdet.

Unsere Analysen können die Forschung zur Verbreitung atypischer Beschäftigung um die kontext- spezifische Verlaufsperspektive bereichern und auf Strukturierungen hinweisen, die gegenwärtig zu wenig wahrgenommen und diskutiert werden. Sie weisen aber auch einige Defizite auf. So lässt es unser Analysedesign bspw. nicht zu, zwischen Altersund Kohorteneffekten zu unterscheiden. Um dies zu realisieren, müsste man über den Zehnjahreszeitraum, der unseren Berechnungen zugrunde liegt, weit hinausgehen, was datentechnisch im Hinblick auf die detaillierte Erfassung atypischer Beschäftigungsformen bislang nicht möglich ist.

Ein weiterer Punkt betrifft die Aussagekraft unserer Ergebnisse für den Diskurs um die Verbreitung und die Determinanten von Prekarisierung. Wir machen lediglich Aussagen über die verlaufsspezifische Einbindung atypischer Beschäftigungsformen. Der Prekarisierungsdiskurs bezieht sich aber auch auf die Wahrnehmung von Unsicherheit und auf Anerkennungsverluste. Unsere Analysen deuten ein Prekaritätsrisiko insbesondere bei Verläufen an, die zwischen Arbeitslosigkeit, Nichterwerb und atypischer Beschäftigung wechseln. Um hier noch anschlussfähiger zu sein, ließen sich in einem nächsten Schritt die Erwerbsverläufe mit Prekaritätsrisiken wie bspw. Armut, Sorgen um den Arbeitsplatzverlust oder Anerkennungsdefizite verknüpfen, um Rückschlüsse darüber zu erhalten, wie kontextspezifisch und sozial ungleich verteilt die Verbreitung von Prekarität ist.

\section{Literatur}

Abbott, A. \& A. Tsay, 2000: Sequence Analysis and Optimal Matching Methods in Sociology: Review and Prospect. Sociological Methods \& Research 29: 3-33.

Aisenbrey, S. \& A.E. Fasang, 2010: New Life for Old Ideas: The "Second Wave" of Sequence Analysis Bringing the "Course" Back into the Life Course. Sociological Methods \& Research 38: 420-462.

Allmendinger, J., 2009: Frauen auf dem Sprung: wie junge Frauen heute leben wollen. Die BRIGITTE-Studie. Bonn: Bundeszentrale für politische Bildung.

Bartelheimer, P., 2011: Unsichere Erwerbsbeteiligung und Prekarität. WSI Mitteilungen 64: 386-393.

Bourdieu, P. (Hrsg.), 1998: Gegenfeuer. Wortmeldungen im Dienste des Widerstands gegen die neoliberale Invasion. Konstanz: UVK.

Brinkmann, U., K. Dörre, S. Röbenack, K. Kraemer \& F. Speidel, 2006: Prekäre Arbeit. Ursachen, Ausmaß, soziale Folgen und subjektive Verarbeitungsformen unsicherer Beschäftigungsverhältnisse. Gutachten für die Friedrich-Ebert-Stiftung. http://library.fes.de/pdf-files/ asfo/03514.pdf (28.4.2010).

Brülle, J., 2013: Unterschiede in den Arbeitsmarktchancen von atypisch Beschäftigten: Effekte von Beschäfti- 
gungsform oder Erwerbspräferenz? Zeitschrift für Soziologie 42: 157-179.

Brzinsky-Fay, C., P. Protsch \& K. Schulze Buschoff, 2007: Atypische Beschäftigung - Umfang, Dynamik und soziale Sicherung im europäischen Vergleich. Unveröffentlichtes Konferenzpapier zur 5. Internationalen ISSA Forschungskonferenz über soziale Sicherheit vom 5. bis 7. März 2007 in Warschau. http://www.issa.int/ ger/Ressourcen/Tagungsberichte/Atypische-Beschaeftigung (19.6.2012).

Brzinsky-Fay, C., U. Kohler \& M. Luniak, 2006: Sequence Analysis with Stata. Stata Journal 6: 435-460.

Bude, H., 2009: Die Spaltung der Gesellschaft, S. 167-182 in: H. König, J. Schmidt \& M. Sicking (Hrsg.), Die Zukunft der Arbeit in Europa: Chancen und Risiken neuer Beschäftigungsverhältnisse. Bielefeld: transcript.

Bukodi, E., E. Ebralidze, P. Schmelzer \& I. Relikowski, 2006: Increasing Flexibility at Labor Market Entry and in the Early Career. A Conceptual Paper for the flexCAREER Project. Working Paper No. 6 (2006). http://www.flexcareer.de/papers/no6.pdf (27.2.2015).

Castel, R. \& K. Dörre (Hrsg.), 2009: Prekarität, Abstieg, Ausgrenzung. Die soziale Frage am Beginn des 21. Jahrhunderts. Frankfurt a.M.: Campus.

Diewald, M. \& M. Eberle, 2003: Unsichere Beschäftigung - unsichere Integration? Auswirkungen destandardisierter Beschäftigung auf die Einbindung in Familie und soziale Netzwerke. Duisburger Beiträge zur soziologischen Forschung No. 1/2003. http://www. ssoar.info/ssoar/files/usbkoeln/2010/579/unsicherebeschaeftigung.pdf (22.11.2011).

Diewald, M., 2004: Thesen zur zukünftigen Entwicklung neuer Formen der Erwerbsarbeit. S. 332-345 in: F. Behringer, A. Bolder, R. Klein, G. Reutter \& A. Seiverth (Hrsg.), Diskontinuierliche Erwerbsbiographien. Zur gesellschaftlichen Konstruktion und Bearbeitung eines normalen Phänomens. Baltmannsweiler: Schneider.

Ebert, A. \& F. Trischler, 2012: Altersübergänge. S. 533-564 in: Forschungsverbund Sozioökonomische Berichterstattung (Hrsg.), Berichterstattung zur sozioökonomischen Entwicklung in Deutschland. Teilhabe im Umbruch. Zweiter Bericht. Wiesbaden: VS.

Fromm, S. \& P. Bartelheimer, 2012: Erwerbsteilhabe. S. 327-358 in: Forschungsverbund Sozioökonomische Berichterstattung (Hrsg.), Berichterstattung zur sozioökonomischen Entwicklung in Deutschland. Teilhabe im Umbruch. Zweiter Bericht. Wiesbaden: VS.

Gensicke, M., A. Herzog-Stein, H. Seifert \& N. Tschersich, 2010: Einmal atypisch, immer atypisch beschäftigt? Mobilitätsprozesse atypischer und normaler Arbeitsverhältnisse im Vergleich. WSI Mittelungen 63: 179-187.

Giesecke, J., 2006: Arbeitsmarktflexibilisierung und soziale Ungleichheit. Sozio-ökonomische Konsequenzen befristeter Beschäftigungsverhältnisse in Deutschland und Großbritannien. Wiesbaden: VS.

Giesecke, J., 2009: Socio-economic Risks of Atypical Employment Relationships: Evidence from the German
Labour Market. European Sociological Review 25: 629-646.

Granovetter, M.S., 1995: Getting a Job. A Study of Contacts and Careers. Chicago: University of Chicago Press.

Grimm, N. \& B. Vogel, 2010: Prekarisierte Erwerbsbiografien und soziale Ungleichheitsdynamik. Perspektiven und Befunde einer qualitativen Panelstudie. In: H.-G. Soeffner (Hrsg.), Unsichere Zeiten. Herausforderungen gesellschaftlicher Transformationen. Verhandlungen des 34.Kongresses der Deutschen Gesellschaft für Soziologie in Jena 2008, CD-Rom. Wiesbaden: VS.

Gundert, S. \& C. Hohendanner, 2013: Do Fixed-Term and Temporary Agency Workers Feel Socially Excluded? Labour Market Integration and Social Well-Being in Germany. Acta Sociologica: 1-18.

Hacket, A., 2012: Erwerbsverläufe in der Haupterwerbsphase. Pluralisierung und Prekarisierung von Erwerbsverläufen? S. 507-532 in: Forschungsverbund Sozioökonomische Berichterstattung (Hrsg.), Berichterstattung zur sozioökonomischen Entwicklung in Deutschland. Teilhabe im Umbruch. Zweiter Bericht. Wiesbaden: VS.

Haisken-DeNew, J.P. \& M. Hahn, 2010: PanelWhiz: Efficient Data Extraction of Complex Panel Data Sets - An Example Using the German SOEP. Journal of Applied Social Science Studies 130: 643-654.

IG Metall Vorstand (Hrsg.), 2012: Schwarzbuch Leiharbeit. Frankfurt a.M.: IG Metall.

Keller, B. \& H. Seifert, 2011: Atypische Beschäftigung und soziale Risiken. Entwicklung, Strukturen, Regulierung. Expertise im Auftrag der Abteilung Wirtschaftsund Sozialpolitik der Friedrich-Ebert-Stiftung. http:// library.fes.de/pdf-files/wiso/08527.pdf (1.12.2011).

Klenner, C. \& T. Schmidt, 2011: Teilzeitarbeit im Lebensverlauf von abhängig beschäftigten Frauen. S. 254-311 in: U. Klammer \& M. Motz (Hrsg.), Neue Wege - Gleiche Chancen. Expertisen zum Ersten Gleichstellungsbericht der Bundesregierung. Wiesbaden: VS.

Klenner, C. \& T. Schmidt, 2012: Minijobs - Eine riskante Beschäftigungsform beim normativen Übergang zum „Adult-Worker-Model“. WSI Mittelungen 65: 22-31.

Kogan, I., 2003: A Study of Employment Careers of Immigrants in Germany. Arbeitspapiere Nr. 66, 2003. Mannheimer Zentrum für Europäische Sozialforschung. http://www.mzes.uni-mannheim.de/publications/wp/wp-66.pdf (22.8.2012).

Kohli, M., 2003: Der institutionalisierte Lebenslauf: ein Blick zurück und nach vorn. S. 525-545 in: J. Allmendinger (Hrsg.), Entstaatlichung und soziale Sicherheit. Verhandlungen des 31. Kongresses der Deutschen Gesellschaft für Soziologie in Leipzig 2002, Teil 1. Opladen: Leske + Budrich.

Lengfeld, H. \& T.-M. Kleiner, 2009: Flexible Beschäftigung und soziale Ungleichheit - Eine Synthese des Stands der Forschung. Zeitschrift für Arbeitsforschung, Arbeitsgestaltung und Arbeitspolitik 18: 46-62.

Mayer, K.U., 1998: Lebensverlauf. S. 438-451 in: B. Schäfers \& W. Zapf (Hrsg.), Handwörterbuch zur Gesellschaft Deutschlands. Opladen: Leske + Budrich. 
Mayer-Ahuja, N., 2003: Wieder dienen lernen? Vom westdeutschen „Normalarbeitsverhältnis“ zu prekärer Beschäftigung seit 1973. Berlin: edition sigma.

Mayer-Ahuja, N., P. Bartelheimer \& J. Kädtler, 2012: Teilhabe im Umbruch - Zur sozioökonomischen Entwicklung Deutschlands. S. 15-40 in: Forschungsverbund Sozioökonomische Berichterstattung (Hrsg.), Berichterstattung zur sozioökonomischen Entwicklung in Deutschland. Teilhabe im Umbruch. Zweiter Bericht. Wiesbaden: VS.

Mewes, J., 2010: Ungleiche Netzwerke - Vernetzte Ungleichheit: Persönliche Beziehungen im Kontext von Bildung und Status. Wiesbaden: VS.

Mückenberger, U., 1985: Die Krise des Normalarbeitsverhältnisses. 1. Teil. Zeitschrift für Sozialreform 31: 415-434.

Mückenberger, U., 2010: Krise des Normalarbeitsverhältnisses - ein Umbauprogramm. Zeitschrift für Sozialreform 56: 403-420.

Oschmiansky, F., A. Mauer \& K. Schulze-Buschoff, 2007: Arbeitsmarktreformen in Deutschland - Zwischen Pfadabhängigkeit und Paradigmenwechsel. WSI-Mitteilungen 60: 291-297.

Rademacher, C. \& P. Ramos Lobato, 2008: „Teufelskreis oder Glücksspirale?" Ungleiche Bewältigung unsicherer Beschäftigung. S. 118-147 in: R. Eickelpasch, P.R. Lobato \& C. Rademacher (Hrsg.), Metamorphosen des Kapitalismus - und seiner Kritik. Wiesbaden: VS.

Sachverständigenrat zur Begutachtung der gesamtwirtschaftlichen Entwicklung (Hrsg.), 2008: Jahresgutachten 2008/2009. Die Finanzkrise meistern - Wachstumskräfte stärken. Wiesbaden: Statistisches Bundesamt.

Sackmann, R. \& M. Wingens, 2001: Theoretische Konzepte des Lebenslaufs: Übergang, Sequenz und Verlauf. S. 17-48 in: R. Sackmann \& M. Wingens (Hrsg.), Strukturen des Lebenslaufs. Übergang - Sequenz - Verlauf. Weinheim, München: Juventa.

Scherer, S. \& J. Brüderl, 2010: Sequenzdatenanalyse. S. 1031-1051 in: C. Wolf \& H. Best (Hrsg.), Handbuch der sozialwissenschaftlichen Datenanalyse. Wiesbaden: VS.

Scherer, S., 2001: Early Career Patterns: A Comparison of Great Britain and West Germany. European Sociological Review 17: 119-144.

Scherschel, K. \& M. Booth, 2012: Aktivierung in die Prekarität: Folgen der Arbeitsmarktpolitik in Deutschland. S. 17-46 in: K. Scherschel, P. Strecheisen \& M. Krenn (Hrsg.), Neue Prekarität. Die Folgen aktivierender Arbeitsmarktpolitik - europäische Länder im Vergleich. Frankfurt a.M.: Campus.
Schmeißer, C., S. Stuth, C. Behrend, R. Budras, L. Hipp, K. Leuze \& J. Giesecke, 2012: Atypische Beschäftigung in Europa 1996 - 2009. WZB Discussion Paper. http://bibliothek.wzb.eu/pdf/2012/p12-001.pdf (23.8.2012).

Schmidt, T., 2012: Junge Erwachsene. S. 469-506 in: Forschungsverbund Sozioökonomische Berichterstattung (Hrsg.), Berichterstattung zur sozioökonomischen Entwicklung in Deutschland. Teilhabe im Umbruch. Zweiter Bericht. Wiesbaden: VS.

Seifert, H., 2005: Was bringen die Hartz-Gesetze? Aus Politik und Zeitgeschichte: 17-24.

Sengenberger, W., 1987: Struktur und Funktionsweise von Arbeitsmärkten. Die Bundesrepublik Deutschland im internationalen Vergleich. Frankfurt a.M.: Campus.

Statistisches Bundesamt, 2008: Atypische Beschäftigung auf dem deutschen Arbeitsmarkt. Begleitmaterial zum Pressegepräch am 9. September 2008 in Frankfurt am Main. https://www.destatis.de/DE/PresseService/Presse/ Pressekonferenzen/2008/Arbeitsmarkt/Pressebroschuere_Arbeitsmarkt.pdf?_blob=publicationFile (28.9.2012).

Statistisches Bundesamt, 2013: Atypische Beschäftigung sinkt 2012 bei insgesamt steigender Erwerbstätigkeit. Pressemitteilung vom 28.08.2013 - 285/13. https://www.destatis.de/DE/PresseService/Presse/Pressemitteilungen/2013/08/PD13_285_132pdf.pdf;jsessionid=334562D25EE9D05F034C92D0B16D382D.cae1? _blob=publicationFile (28.10.2013).

Vogel, B., 2006: Sicher - Prekär. S. 73-92 in: S. Lessenich \& F. Nullmeier (Hrsg.), Deutschland. Eine gespaltene Gesellschaft. Bonn: BPB.

Völker, S., 2009: ,Entsicherte Verhältnisse“ - Impulse des Prekarisierungsdiskurses für eine geschlechtersoziologische Zeitdiagnose. S. 268-286 in: B. Aulenbacher \& A. Wetterer (Hrsg.), Arbeit. Perspektiven und Diagnosen der Geschlechterforschung. Forum Frauen- und Geschlechterforschung. Band 25. Münster: Westfälisches Dampfboot.

Wagner, G.G., J.R. Frick \& J. Schupp, 2007: The German Socio-Economic Panel Study (SOEP) - Scope, Evolution and Enhancements. Schmollers Jahrbuch 127: 139-170.

Weinkopf, C., B. Hieming \& L. Mesaros, 2009: Prekäre Beschäftigung. Expertise für die SPD-Landtagsfraktion NRW. http://www.iaq.uni-due.de/aktuell/veroeff/2009/ weinkopf_Prekaere_Beschaeftigung.pdf (24.11.2011). 


\section{Autorenvorstellung}

Petra Böhnke, geb. 1969 in Lüneburg. Studium der Soziologie, Germanistik und Politikwissenschaft in Göttingen, London und Berlin. Seit 2011 Professorin für Soziologie, insbesondere Soziologie des sozialen Wandels an der Universität Hamburg.

Forschungsschwerpunkte: Wandel sozialer Ungleichheit und Sozialstrukturanalyse im wohlfahrtsstaatlichen Vergleich zu den Themen: Armut, soziale Mobilität, intergenerationale Transmission, soziale Integration, Sozialkapital, Lebensqualität.

Wichtigste Publikationen: Subjektive Umgangsformen mit prekärer Erwerbsarbeit - Zwischen Orientierung an und Ablösung von der Normalarbeitsgesellschaft (mit A. Gefken \& F. Stockem), in: Berliner Journal für Soziologie 2015; Die intergenerationale Weitergabe von Armut bei MigrantInnen zweiter Generation (mit B. Heizmann), in: H. Weiss et al. (Hrsg.), Zwischen den Generationen, Wiesbaden 2014; Implications of the Activation Paradigm on Poverty and Social Exclusion in Germany: Facts, Hypotheses, Uncertainties, in: German Policy Studies 2010; Are the Poor Socially Integrated? The Link Between Poverty and Social Support in Different Welfare Regimes, in: Journal of European Social Policy 2008.

Janina Zeh, geb. 1984 in Esslingen a.N. Studium der Soziologie und Erziehungswissenschaften in Leipzig. Seit 2011 wissenschaftliche Mitarbeiterin an der Universität Hamburg.

Forschungsschwerpunkte: Prekarisierung von Erwerbsarbeit, Normalitätsvorstellungen von Erwerbsarbeit und wohlfahrtsstaatlicher Absicherung, Genderungleichheiten, freiwilliges Engagement.

Wichtigste Publikationen: Exklusion: Ursprung, Debatten, Probleme, in: Heinrich-Böll-Stiftung (Hrsg.), Inklusion: Wege in die Teilhabegesellschaft, Frankfurt a.M. 2015; Apportionment Methods (mit U. Kohler), in: Stata Journal 2012.

Sebastian Link, geb. 1986 in Koblenz. Studium der Soziologie, Volkswirtschaftslehre und Politikwissenschaft an den Universitäten Hamburg, Bonn und Genf. Seit 2013 wissenschaftlicher Mitarbeiter an der Universität Hamburg.

Forschungsschwerpunkte: Armuts- und soziale Ungleichheitsforschung, Sozialkapital und intergenerationalen Beziehungen aus Lebenslaufperspektive. 